\title{
Vicente Noguera et son Discours sur la langue et les auteurs d'Espagne.
}

L'érudit portugais, fort oublić et à tort, sur lequel je voudrais ramener l'attention de tous ceux qui s'intéressent à l'histoire littéraire de la péninsule ibérique, ne se présente pas à la postérité, il faut le reconnaitre, avec un bien lourd bagage d'œuvres originales. Esprit plus critique que créateur, plus apte à s'assimiler les travaux d'autrui et à les juger qu'à découvrir des terres inconnues et à étendre l'horizon de la science, cet amateur distingué n'a pas composé un ouvrage qui permette de le classer parmi les écrivains ou les savants de son pays; et pourtant, comme j'espère le montrer, son nom mérite de vivre, car c'est quelque chose que d'avoir été l'instigateur et le protecteur de plusieurs entreprises scientifiques d'une réelle importance, d'avoir contribué par sa situation sociale et ses relations à la culture des lettres nationales et à leur diffusion à l'étranger. Ce fut un Mécène, mais un de ces Mécènes intelligents et sérieusement aficionados, dont les écus seuls ne font pas la gloire. Vicente Noguera intervenait directement dans les travaux qu'il jugeait dignes de son appui, et les documents qui vont être produits établissent sa compétence et font pressentir ce qu'il aurait pu livrer à la publicité, s'il avait su sortir de son rôle de protecteur et de critique. Mais n'est-ce pas à l'accident quelque peu mystérieux, qui troubla la vie de cet homme si fortement préparé et l'obligea à quitter brusquement sa patrie, qu'on doit attribuer cette regrettable improduction? Je ne le pense pas, car le talent vraiment créateur sait lutter contre des obstacles de cette nature, et si Noguera avait eu la volonté de produire des ceuvres originales, son séjour forcé à Rome dans l'intimité d'hommes de lettres et de savants tels que le cardinal Francesco Barberino, Lucas Holstenius et le R. P. Dupuy n'eùt guère pu nuire à la divulgation de ses idées et de ses recherches.

Ici je n'essaye pas une biographie de Vicente Noguera. Les lettres et autres documents que j'ai trouvés éclairent, il est vrai, d'un jour assez vif certaines périodes de son existence et certains côtés de son esprit, mais bien des circonstances de sa vie restent encore couvertes d'un voile qu'il n'est pas en mon pouvoir de lever pour le moment. Néanmoins comme tous ces témoignages se tiennent l'un l'autre d'assez près, s'éclairent mutuellement et découvrent 
au moins quelques parties du sujet, je n'ai pas cru devoir en retarder la publication. Puisse la simple coordination de ces notes stimuler quelque érudit portugais ou espagnol à écrire une véritable biographie de ce personnage curieux et attachant. De bons matériaux existent certainement encore dans plusieurs bibliothèques péninsulaires: il s'agit de les rechercher et de les mettre en cuuvre.

Parmi les documents que j'ai eu la chance de retrouver, il en est un d'une valeur particulière, qu'il importait de détacher des autres et d'entourer de quelques soins: je veux parler du Discours sur la langue et les auteurs d'Espagne. Ce morceau d'histoire et de critique littéraire, travail de circonstance rédigé à la hâte, ne donne pas, j'en conviens, toute la mesure du savoir et du talent de notre portugais; cependant il est assez long et détaillé pour qu'on puisse, en le lisant, se faire une idée nette de la tournure d'esprit, des aptitudes critiques et du goût littéraire de son auteur. Il m'a donc paru convenable de le produire en dernier lieu, avec une glose de ma façon, pour servir de couronnement aux notes biographiques dont j'ai tenté de former un petit faisceau.

Quelque oublié qu'il ait été après sa mort par ses compatriotes, ${ }^{1}$ Noguera a pourtant mérité l'attention de trois chercheurs, qui ont groupé autour de son nom divers renseignements fort utiles. Pour procéder méthodiquement, je commencerai par les transcrire. Voici d'abord l'article inséré par le premier bibliographe portugais, Joaõ Soarez de Brito, dans son Theatrum Lusitaniae literarium sive Bibliotheca scriptorum omnium Lusitanorum ${ }^{2}$ : „D. Vincentius Nogueyra, patria Olissiponensis, egregius, i-e. regius senator et Archiducis Leopoldi cubicularius, utrique Majestati Cæsareæ et Catholicæ consiliarius et Utriusque Signaturæ referendarius, vir eruditus et linguarum exoticarum peritia clarus. Scripsit Summam Historice Lusitane et Notas marginales apposuit Didaco de Mendoça, Belli Granatensis autori, quem excudi curavit, et alia. Obiit Romæ, quo ipsum sua fata duxere. - D. Franciscus Eman. in Epist. cit." 3

Diogo Barbosa Machado, qui écrivait près d'un siècle plus tard, a beaucoup augmenté la notice de son prédécesseur ${ }^{4}$ : „D.Vicente Nogueira, naceo em Lisboa no anno de 1586, sendo filho do Doutor Francisco Nogueira, cavalleiro do habito de Saó-Tiago, desembargador da Casa da Suplicaçaõ e juiz da Coroa, e do Conselho de Estado de Portugal. Quando contava doze annos o fez ElRey moço fidalgo, e tal era o talento que mostrou em idade

1 Par compatriotes j'entends les Espagnols aussi bien que les Portugais. A l'époque de Noguera lc Portugal était uni politiquement à l'Espagne, et au point de vue littéraire et scientifique les deux nations n'en font qu'une.

2 Cet ouvrage n'a pas été imprimé, mais Barbosa Machado l'a fortement mis à contribution. L'exemplaire ms. qu'en possède la Bibliothèque Nationale, et qui semble avoir été préparé pour l'impression, porte ces lieu et dates: „Conimbricæ, typis academicis, anno christiano MDCLV, a restauratione Lusitani Imperii XV. Cum facultate Superiorum."

3 Bibl. Nat. Nouv. acq. lat. 1290, p. 291.

+ Bibliotheca lusitana, t. III, Lisbonne, 1752, p. $784-785$. 
taõ tenra que contrahio grande amizade com o Condestavel de Castilla, D. Bernardino de Mendoça e o Duque de Feria. Estudada filosofia se graduou na facultade de Canones, e depois foy senador da Casa da Suplicaçao, de que tomou posse a i3 de março de I6I3, e conego da cathedral de Lisboa. Soube com perfeiçað as lingoas latina, grega, caldaica, syriaca, arabica, italiana, franceza e castelhana. Teve bastante instruçao da historia sagrada e profana, como tambem da poezia, mathematica, musica e algebra. Sahindo involuntariamente da patria no anno de 1631 , experimentou fóra della a fortuna mais propicia assim nos lugares que teve, como nas estimaçoens que alcançou das pessoas da primeira jerarchia, sendo senhor de Rios frios, referendario de ambas as signaturas em Roma, conselheiro da Magestade Catholica e Cesarea e camarista da chave dourada do archiduque de Austria Leopoldo. Falleceo em Roma no palacio do cardeal Francisco Barberino, vice cancellario da Igreja Romana em o anno de 1654, quando contava 68 annos de idade. Sobre a sua sepultura se lhe gravou o seguinte epitafio:

Vincentio Nogueira Ulyssiponensi

Hereditario in Rios frios Domino

Utriusque Signatura

In Romana Curia Referendario;

Casarece Catholicaque Maiestutis

$A$ Consiliis;

Leopoldi Austrice Archiducis

Clavis aurece cubiculario.

Animo forti in adversa fortuna,

Moderato in secunda;

Liberalium artium

\& linguarum etiam Orientalium

Peritissimo,

Profusa in pauperes pietate,

Miagnificentia in amicos singulari,

M. Antonius de Nobilibus Bononiensis

Grati animi monumentum posuit.

Diversos escritores lhe celebrarao o seu nome buscandoo para Mecenas das suas obras, como forao Zacuto Lusitano Praxis Hist. Med. Tom. 7, Bento Gil de Privilegiis advocatorum, Luiz Tribaldos na Dedicatoria da Guerra de Granada, composta por Diogo de Mendoça. Gabriel Pereira de Castro, Decis. 97, o intitula peritissimum; Joan Soar. de Brito, Theatr. Lusit. Litter. lit. V, n. 22, Vir sruditus peritia linguarum exoticarum ${ }^{1}$; Barthol., Bibl. Rabin., Tom. 2, p. 809, in hebraica lingua admodum versatus. D. Franc. Manoel, Cart. dos Author. Portug. que he a I da 4 cent. das suas Cartas, e Lopo Felix da Vega, Laurel de Appollo, Sylv. 3:

1 Cf. plus haut, p. 2. 
Don Vicente Noguera

Tuviera asiento entre latinos grave,

Laurel entre toscanos

Palma entre castellanos,

Por la dulzura del hablar suave,

$\mathrm{Y}$ entre franceses $\mathrm{y}$ alemanes fuera

Florida primavera,

Que como ella de tantas diferencias

De alegres flores se compone y viste,

Asi de varias lenguas y de ciencias,

En que la docta erudicion consiste.

$¿$ Qué libro se escribió que no le viese?

$¿$ Qué ingenio floreció que no le honrase?

$¿$ En qué lengua se habló que no supiese?

¿Qué ciencia se inventó que no alcanzase?

¡Oh musas castellanas y latinas,

Francesas, alemanas y toscanas,

Coronad las riberas lusitanas

De lirios, arrayanes y boninas;

No quede en vuestras fuentes cristalinas

Laurel que en ellas su hermosura mire,

Donde Dafne amorosa no suspire

Por no bajar á coronar la frente

Compoz:

Deste, de todos vencedor, Vicente! I

Carta escrita de Lisboa a 28 de setembro de 1615 a Jacolio Augusto Thuano, presidente do Senado de Pariz........

Relaçoens tiradas de varios papeis para la historia del Rey D. Sibastiad com as noticias de Francisco Giraldes em Roma e Inglaterra $e$ de Lourenço Pires de Tavora em Roma, escritas por Vicente Nogutira em Lisboa, a 12 de setembro de 1618, fol. Ms. Conservaó-se na livraria do real convento de Thomar da ordem militar do Christo.“ ?

Le troisième érudit, auquel on doit d'avoir insisté quelque peu sur la personne de notre portugais, est l'éminent helléniste Jean François Boissonade, qui a consacré à Noguera une note de son édition des lettres de Lucas Holstenius ${ }^{3}:$ „V. Noguere, cujus memi-

- Dans le Catálogso de los autores citados en el Laurel de Apolo de la Coleccion escogida de obras no dramaticas de Frey Lope Félix de Veya ('arpio (Bibl. Rivadeneyra), le compilateur, D. Cayetano Rosell, commente ainsi le passage cité: „Noguera (D. Vicente), portugués“. C'est peu compromettant.

2 Peut-être existe-t-il quelque autre notice sur Noguera dans l'une ou l'autre des bibliographies portugaises manuscrites citées par Petzholdt, bibliotheca bibliographica, Leipzig 1866, p. 380; toutefois comme le Diccionario bibliographico portuguez d'Innocencio Francisco da Silva n'a pas d'article consacré à notre auteur, j'en conclus que le savant bibliographe n'a rien trouvé a ajouter a Barbosa Machado.

3 Luca Holstenii epistola ad diversos, quas ex editis et ineditis codicibus collegit atque illustravit Fo. Franc. Boissonade. Parisiis 1817, in 80, p. 297. La note se rapporte à un passage d'une lettre de Holstenius à Peiresc (Rome, 7 mars 1637), où il est parlé de Noguera. 
nerunt Noster ad calcem Fpist. XI.VII 1; Gassendius V. Peir., p. I96, illum vocans Lusitanum nobilem; et Is. Vossius in Syll. Burman. t. III, 1). 627, cui eques Iusitanus, vir probus doctusque dicitur. Inter Judicia post Thuani Historiam, t. VII, p. 82, legenda est longa epistola Vincentii Nogueyra, nam sic nomen effert ipse, ubi multa super se narrat studiisque suis. De Cantabrica, ut videtur, lingua exstabant literae Peirescii ad Nogueyram, teste Buchardo in Laudatione Peirescii, p. 272. Illi praefationem Quaesitorum per epistolas Fort. Licetus inscripsit. Plura forte de hoc docto viro praebuit Barbosae Bibliotheca Lusitana qua careo."

A l'aide de tous les témoignages qui viennent d'être énumérés ot de ce que j'ai pu y joindre, je vais essayer maintenant de plinter quelques jalons dans ce terrain peu défriché.

Vicente Noguera 2 , nć en 1586, ¿̀ Lisbonne, appartenait à une famille d'origine moitié portugaise, moitié castillane ${ }^{3}$, établie au Portugal, où elle possédait la petite seigneurie de Rios Frios. ${ }^{4}$ Son père, le docteur en droit Francisco Noguera, après quelques années passées à l'université de Coimbre, où il se distingua autant par sa science que par sa grande modestie, eut à remplir d'importantes fonctions administratives et judiciaires. Il fut desembargador (conseiller) da Casa da Suplicaçaõ et juge de la Couronne (juiz da Coroa). Plus tard l'annexion de sa patrie à l'Espagne l'éleva à une charge plus considérable encore, celle de membre du Conseil d'Etat de Portugal, créé par Philippe II aussitôt après la conquête, ce qui l'obligea à venir résider à Madrid, où se fit l'éduçation de ses deux fils, Paulo Alfonso, l'aînć, et Vicente. De l'aîné nous savons seulement, par I.uis 'Tribaldos (l. c.), qu'après avoir suivi pendant un certain temps, quoique n'étant pas juriste (siendo de capa l' espada), la carrière administrative, sa médiocre ambition ou "quelque autre cause“ l'amena à renoncer à ses fonctions publiques pour vivre en célibataire des revenus d'une commanderie de $\therefore$ Jacques qu'il avait héritée de son père.

D. Vicente, qui va maintenant nous occuper exclusivement, a pris soin de raconter lui-même les péripéties de son existence jus-

1 Lettre de Holstenius à Fortunio Liceto, Rome, 2 avril 1638, Ibid., p. 300.

2 Cette forme Noguera, que j'ai partout adoptée dans ce mémoire, est autorisée en premier lieu par D. Vicente lui-méme, qui a signé Noguera sa lettre à Peiresc du 27 janvier 1637 (voy. plus bas), seul document autographe que je connaisse de lui; puis par Luis Tribaldos de Toledo (Dedicatoria de la Guerra de Granada, Lisbonne 1627): „La familia Noguera, ó Nogueira, como la llaman en esse reino [de Portugal]." Noguera est la forme castillane, Vogueira la variante portugaise d'un nom assez répandu dans la Péninsule.

3 ,,Mis padres y abuelos fueron unos de Castilla, otros de Portugal, adonde fixó raices esta familia de los Nogueyras." Lettre de D. Vicente à De Thou; voy. plus bas.

4 Dans sa Dedicatoria de la Guerra de Granada Luis Tribaldos de Toledo a inséré une généalogie de la famille Noguera, dont les éléments lui ont probablement été fournis par $D$. Vicente, et qui doit par suite présenter des garanties sérieuses d'exactitude. J'y renvoie les personnes désireuses de connaitre en détail l'histoire des ascendants de notre auteur. 
qu'à l'âge de vingt-neuf ans dans la curieuse lettre qu'il écrivit de Lisbonne à l'illustre historien Jacques Auguste de Thou, le 28 septembre 1615. Alors dans tout le feu de ses études, il trouve un plaisir singulier à détailler son acquit scientifique au célèbre écrivain, dont il espérait devenir le correspondant régulier pour les choses d'Espagne et de Portugal. De ce que la lettre commence par un éloge en forme des Histoires, on pourrait conclure que de Thou avait envoyé à l'érudit portugais un exemplaire des livres publiés de son grand ouvrage, lui demandant en retour une sorte d'autobiographie et des renseignements sur l'état des lettres et des sciences dans la Péninsule; jusqu'ici cependant je n'ai pu découvrir aucune trace de rapports épistolaires entre les deux érudits antérieurement à cette lettre de Noguera, dont voici la teneur:

\section{Al presidente Jac. Augusto Thuano. ${ }^{1}$}

Si dixere á V. S. que no he leido mejor historia que la suya (con ser medianamente versado en todas) ni aun otra que en mi juicio se le yguale, no sera lisonja ni encarecimiento, y qualquiera que con cuydado la mirare, entendera bien que pueda ser idea ó espejo al qual se ayan de examinar las que adelante salieren, tan justamente como con los preceptos de Luciano. Alábola por mil titulos, mas principalmente por la verdad, en la qual V. S. se desnuda de todos los affectos y la sigue con tanta libertad, que á vezes podra escandalizarse quien tuviere en su animo qualquiera pequeño prejuizio; pero los venideros, en quien ya estuvieren muertas ó á lo menos mas alexadas las passiones, sin falta la alabarán ${ }^{2}$ como $^{3}$ merece. Lo que aqui condenan algunos, que es no ensangrentarse V. S. mucho la espada contra los sectarios y alaballes sus virtudes, quando las tienen notorias, no merece respuesta; pues V. S., como cavallero catholico y pio, vitupera sus dogmas sufficientemente, y ellos son los que lo merecen: que las personas, quanto mejor las trataremos de obras y de palabras, tanto mas las havremos dispuesto para la conversion de sus yerros á la fee orthodoxa de la sancta yglesia romana, nuestra madre, que es lo que solo de ellos pretendemos. Punto, que si se huviera ponderado en Castilla, no castraran en V. S. lugares muy sufrideros. ${ }^{4}$ Concluyo, Señor, este punto con que la tengo por la mas verdadera historia de las humanas. Pássome á la frasis y diccion, y digo que con razon pudiera competille lo que ya se dixo de la de Plauto, porque no puede haverla mas propria, pura y natural, y suma elegancia, sin afectarla, con lindissimos colores en qualquiera descripcion y tan llena de conceptos y sentencias, sin echarse de ver (que es lo que yo mucho admiro), que quien huviere leydo una plana, distilará mas sentencias y aforismos que de Sallustio ó Tacito. - Demas de lo dicho, merece suma alabança una cosa nueva pero convenientissima, y es, á la entrada de los successos de cada reyno, una muy puntual descripcion de sus confines, qualidades, formas y mudanças de govierno, bastantes á instruir

' Cette lettre, imprimée et traduite dans les Historiarum, éd. de Londres, 1733, t. VII, VIe partie, p. 82-85, ne fut pas adressée directement au savant français mais à un personnage portugais ou espagnol, résidant à Anvers, „le Cav. Chimenez", qui l'envoya à de Thou. De la lettre de Noguera il existe aussi une copie manuscrite à la Bibliothèque Nationale, Collection Dupuy, vol. 409 , ff. $50-5^{2}$, qui porte cette indication: „Receue a Poitiers, le 10 janvier 1616".

2 Ms. Dupuy : „celebrarán“.

3 Ms. Dupuy: "como ella“.

- La liste des passages des Historiarum mis à l'index par l'Inquisition espagnole a été insérée au tome VII de l'édition citée, partie I, p. 63 à 137 . 
á quien la ${ }^{1}$ leyere para poder hablar y juzgar de ellas. Pues el amor que V. S. muestra á las letras y sus professore's no merece silencio, antes grandes encomios (quales yo hiziera, y justamente, á ser esto panegyrico y no carta, que ya no es larga, si bien corta, cn respecto de lo que yo amo y estimo á V. S.); $\mathrm{le}^{2}$ suplico muy encarecidamente quiera conocerme por grande amigo y servidor suyo, conciliado por sus merecimientos, y mandarme como á tal ${ }^{3}$ lo que de su gusto se offreciere en Hespaña y señaladamente en Portugal, adonde oy quedo de assiento en la ciudad de Lisboa, sirviendo á S. Mg. en cl Consejo Supremo de las causas civiles y criminales, llamado de la Suplicacion; $y$ porque, aunque en esta corte $y$ en otras me conocen, en essa no hallará $\dot{v}$. S. quien de mi le diga, lo hare yo, haziendome muchas fuerças á mi modestia y fingiendome necessitado para yr contra mi costumbre.

Mis padres y abuelos fueron unos de Castilla, otros de Portugal, adonde tixó rayzes esta familia de los Nogueyras. Mi padre, chefe de ella, fue persona de raras letras y santidad, que le subieron al Consejo de Estado de Portugal, que assiste à la persona del Rey, en el año de 1598, y alli murio en I612. Nacy en 1586, y de doze años supe bien latin, edad en la qual me tomó S. Md por moço-fidalgo suyo, que es insignia de la primera classe de la nobleza. Los inviernos cursava en las universidades de Alcalá, Valladolid, Salamanca, y los veranos en el palacio, no intermittiendo con el curso de la Corte los estudios, antes aumentandolos con el trato y familiaridad de aquellos grandes ministros, que me admittian, siendo tan moço, á sus platicas mayores, pronosticandome grandes suficiencias y fabricandose grandes esperanças de mi talento. Eran estos cl Condestable 4 , mi señor (que me honrava por pariente con honras publicas y en actos regios), el conde de Miranda ${ }^{5}$, el de Chinchon ${ }^{6}$, D. Bernardino de Mendoça ${ }^{7}$, el duque

1 Ms. Dupuy: „las““.

2 Ms. Dupuy: "’á quien“.

3 Ms. Dupuy: "tal en todo".

- D. Juan Fernandez de Velasco, sixième connétable de Castille, cinquième duc de Frias, etc. Un des hommes les plus remarquables de son temps. Gouverneur du Milanais à la fin du règne de Philippe II, commandant des forces espagnoles en Franche-Comté en 1595, où il eut l'honneur de combattre contre Henri IV, membre du Conseil d'Etat en 1599, plus tard président du Conseil d'Italie, ambassadeur en Angleterre pour y traiter de la paix avec Jacques Ier, nommé une seconde fois gouverneur du Milanais en 1610, ce grand personnage mourut le 23 mars 1613 (voy. Luis Cabrera de Córdoba, Relaciones de las cosas sucedidas en la córte de España, deste 1599 hasta 1614 , Madrid 1857 , p. 513). Ce fut aussi un littérateur et un savant. On a de lui une critique erudite des commentaires de Hernando de Herrera sur Garcilasu (publ. par la Sociedad de biblisfilos andaluces, t. II de la Ire série) et deux discours sur la prétendue venue de S. Jacques en Espagne (impr. à Valladolid en 1605). Il réunit une splendide bibliothèque, dont le catalogue, malheureusement incomplet, est conservé à la Bibliothèque Nationale de Madrid (V-156). Pour plus de détails voy. Matias de Novoa, Historia de Felipe IIY, t. I, p. 209 à 218 (Coleccion de doc. inéd. para la hist. de España, t. LX) et Lopez de Mendiçorroz, Observaciones de la vida del Condestable Fuan Fernandez de Velasco y cifra de sus dictamenes. Vigeven $1625,4^{\circ}$.

5 D. Juan de Zuñiga y Avellaneda, premier duc de Peñaranda, sixième comte de Miranda. Fut vice-roi de Catalogne et de Naples, président des conseils d'Italie et de Castille, membre du Conseil d'Etat de Philippe II et de Philippe III. Mourut le 4 septembre I608. Voy. Cabrera, l. c., p. 349 et Garma, Teatro universal de España, Barcelone I75I, t. IV, p. 65 et 26I.

6 D. Diego de Cabrera y Bobadilla, troisième comte de Chinchon, trésorier général de la couronne d'Aragon, membre du Conseil d'Etat de Philippe II et de Philippe III. Mourut le 23 septembre I608. Voy. Garma, l, c., p. $6 \mathrm{r}$.

7 D. Bernardino de Mendoza, fils cadet d'Alonso Suarez de Mendoza, troisième comte de Coruña. Chevalier de $\mathrm{S}$. Jacques, ambassadeur en France 
de Feria. ${ }^{1}$ Con esta aprobacion, llegando á veinte cinco años, me hizo S. Mu su consejero en lil Suplicacion, despacho que, si bien grandissimo y en que nadie ha entrado con menos de 30 años, que aun oy no tengo, me cortó el hilo á medras y lugares muy aventajados, que yo verisimilmente alcançara, á no entrar por estos oficios literarios, con que van adelante los mas antiguos. Despues de oida philosophia, me gradué en canones y leyes, y siempre me di con tanta curiosidad á las letras humanas que para solo la lengua griega truxe de Roma á Constantino Sophia Smirnes, doctor en theologia y colegial del Collegio Griego, y le tuve en mi casa cinco años, en los quales passamos quasi todos los auctores con un profundo estudio $y$ algunos de la primera letra hasta la ultima, Homero, Herodoto, Platone, Thucydides, etc. La hebrea sé con la misma suficiencia que la materna. La chaldca y arabica medianamente. Italiana y francesa bien, menos bien la thudesca. De historia es increible quanto he leydo de generales, particulares, chronicas, geographias, etc. En las mathematicas hago estudio firmado, siendo bien instruido en ellas, sacando la theoria de la musica. Y lo que mas procuro es la algebra, en la qual todo lo he manejado salvo las obras de Vieta, trayendo un excelentissimo maestro de ella de Marruecos, corte del Xarrife, adonde la enseñava por un modo arabigo aventajado al nuestro. Y para me dar con mas quietud á las letras, me hize clerigo ${ }^{2}$, haviendo alcanzado beneficios y pensiones con que filosofar. La mayor falta que siento es no haver peregrinado, pero alcanzando licencia que tengo pedida, me tendra V. S. en su museo á aprender y oyer (sic) de esse oraculo de prudencia, mas benemerito á que vayan de España á buscarle que á Tito Livio. Lo que suplico á V. S. afectuosamente es el silencio $^{3}$ de todo lo referido, que me averguençaré mucho de que se sepa que hablo algo de mis cosas, aun siendo ellas tan notorias. Y si V. S. se cansare de tanta lectura, perdóneme el travajo, pues la causa ha sido mostrarle que tiene razones y motivos para ponerme en la lista de sus mayores amigos, y que, si como nacy de los Pyreneos aca, huviera nacido ${ }^{4}$ de ellos alla, para tener nombre me faltara poco; pero contentaréme con que V. S. lo sepa para escrivirme aqui á Lisboa, mandando echar la carta en el ordinario con el sobreescrito para mi. E guarde Dios a V. S. como deseo. Lisboa, 28 de setiembre de I6I5.

\section{Don Vincente Nogueyra.}

De Thou répondit à ces avances et ces offres de service par une longue lettre ${ }^{5}$, dont nous ne reproduirons que quelques passages, car l'érudit français y traite surtout de son histoire, des circonstances politiques au milieu desquelles il l'a composée et des ennemis que son impartialité lui a créés: toutes choses qui ne rentrent pas dans notre sujet. En commençant il expose les motifs qui l'ont poussé à répondre en latin et témoigne la vive satisfaction qu'il a éprouvée à recevoir d'au delà les monts des compliments si flatteurs:

et en Angleterre, auteur estimé des Comentarios de lo sucedido en las gruerras de los Paises Bajos, desile el año 1567 hasta el de 1577. Mourut en I604. Voy. Lopez de Haro, Nobiliario genealígico, t. I, p. 40\%, et Cabrera, I. c., p. 225 .

1 D. Lorenzo Suarez de Figueroa, deuxième duc de Feria, qui joua, comme agent de Philippe II, un rôle si important à Paris à la fin de la Ligue.

2 Noguera fut élevé à la dignité de chanoine de la cathédrale de İisbonne; voy. la notice de Barbosa Machado.

3 Ms. Dupuy: ,secreto“.

4 Ms. Dupuy: "naciera“.

5 Imprimée dans les Historiarum, éd. de Londres 1733, t. VII, part. VI, p. 85-87. Le volume cité de la collection Dupuy en contient aussi une copie manuscrite, ff. $54-57$. 
„Humanissimis litteris tuis, V. C., quaw Pictavii in comitatu regio Burdigala rediens ${ }^{\prime}$ ante mensem accepi, respon surus, diu dubitavi gallicene (nam te vernaculam nostram callere significas) an communi omnium lingua ad te scriberem. Nam, ut multo mihi commodius erat gallice ad te scribere ${ }^{2}$, ita verebar, ne si litteræ in manus alienas venirent, aut ignoratione linguæ secus quam oporteat acciperentur, aut malignum interpretem nactæ, facilius in pravum sensum per calumniam detorquerentur. Itaque expeditius visum, ut latine ad te scriberem; et tibi pro tam obvia humanitate gratias quam possem maximas agerem. Nam quid iucundius, quid honorificentius mihi accidere potuit, delata tam benevole ${ }^{3}$ amicitia tua? Quid præter expectationem magis, quam Celtam hominem ab Hispano non solum tam egregiis laudibus supra meritum ornari, sed etiam haut dubio victricis post invidiam domitam fortunac augurio in posterum impleri? Scis quantie qualesque Gallo-Francis cum Hispanis centum amplius abhinc annis emulationes intercesserint, quæ per bella continua inter eos in odium plus quam civile vulgo exarserunt. Sed virtus, ut gratiam, sic et odium nescit, omnique pravo affectu spoliata, in id unum yuod iustum et honestum est, nullo Graeci Scythave discrimine, recta fertur; totumque orbem terrarum animo pervagata, nullo maris tractu, nullis montibus aut fluviis, quos aut natura inter populos pro limite posuit, aut reges finiendis inter vicinos bellis pacti sunt, discluditur. Vel insigne in te ipso exemplum vide. Tu medio Pyrenco in ultima Lusitania natus, hominem Gallum, non in proxima Aquitania, sed longe in extrema Celtica ad Sequanæ ripam positum, virtutis opinione conciliare tibi voluisti, affatu tuo benigne dignatus es, eiusque in Hispania tua, ubi tantopere exagitatur, patrocinium, non rogatus, suscepisti“, etc.

De Thou accepte insuite les propositions de Noguera et termine en lui demandant les dates de la mort et les éloges de divers Kspagnols et Portugais célèbres:

„Peto igitur a te, vir clarissime, loco summi beneficii, ut emortualeis dies Joannis Barosii vestri, qui Indica scripsit, Petri Nonii, praestantissimi mathematici, Amati item excellentis medici, Petri Stellæ Franciscani, ... 'Alcantaræ, et huiuscemodi vestratum philologorum elogia, quæ reperire poteris, cum lie obitus diligenter notato, ad me per otium mittas; nam vereor ut qua de Barosio et Nonio scripsi satis certa sint, et si quidem vera sint, cupio de iis a te certior fieri. Vide quam familiariter tecum agam, postquam semel ad amicitian tuam mihi aditum tam humane patefecisti ... Julioduni 5, in conventu quo ad motus nostros componendos a rege cum illustrissimis comite Brissaco E. T. et Villaregio missus sum. Pridie Kal. Mart. anni bissextilis MDCXVI.“

Dans sa lettre à de Thou Noguera semble indiquer qu'il renonça à sa charge de conseiller en prenant possession de son canonicat, bonne sinécure, qui allait lui donner le temps de poursuivre se's études favorites, et le moyen de protéger efficacement les savants dont il savait apprécier le mérite. Je crois toutefois qu'il cumula jusqu'en 16 I8 ses fonctions politiques et sa place de

1 La Cour arriva à Poiticrs le 6 janvier 1616 et en repartit le 2 i du mème mois. Voy. les Mémoires de P. Phely'peaux de Pontchartrain (Vouz'elle iollection de Michaud et Poujoulat, Deuxic̀mc série, t. V, p. 357 et 358).

2 L'auteur des Historiarum trouver beaucoup plus facile d'écrire en français qu'en latin, n'est-ce pas singulier?

3 Ms. Dupuy: ,prolixe“.

4 Sic dans l'imprimé et la copie. Je suppose que de Thou a voulu parler de S. Pierre d'Alcántara.

5 Loudun, où de Thou, le maréchal de Brissac, Nicolas de Neufville de Villeroi et d'autres personnages s'étaient rendus le 30 février 1616 de la part du roi Louis XIII pour y traiter de la paix avec le parti du prince de Condé. Voy. le P. Daniel, Histoire de France, t. XIII, p. 126. 
chanoine; autrement le panégyriste, Luis Tribaldos de Toledo, n'aurait pas pu dire de son Mécène "qu'il avait pratiqué six ans de suite" 1 la jurisprudence au Conseil de la Supplication, où nous savons qu'il fut appelé en I6I 2 à l'âge de vingt-cinq ans. C'est pendant ces six ans passés à Lisbonne ${ }^{2}$ que Noguera rédigea le travail historique sur le roi Sébastien, dont le titre, cité par Barbosa Nachado, indique plutôt un recueil de matériaux qu'une relation suivie. A l'époque de l'auteur de la Bibliotheca Lusitana cette compilation manuscrite, datée de Lisbonne, le I 2 septembre I618, était conservée au monastère de Tomar de l'ordre militaire du Christ. Depuis lors qu'est-elle .devenue? Les érudits portugais répondront, je l'espère.

Si l'on s'en tient, comme il convient de le faire, aux renseignements biographiques de Luis Tribaldos, ce fut à la fin de cette année 1618 ou l'année suivante ${ }^{3}$ que Noguera vint à Madrid présenter sa démission de conseiller du Roi, afin, dit son protégé, de „vaquer exclusivement aux lettres et aux hautes études“. Après avoir ainsi librement renoncé à ses fonctions publiques, D. Vicente courut s'enfermer dans son musée, qu'il appelait son recoin (rincon), et reprendre avec amour les études littéraires et scientifiques, dont il traçait naguères le plan à de Thou.

En 1625 on voit apparaître son nom sur le titre d'un recueil de poésies, les Obras de Francisco de Figueroa, Laureado Pindaro hespañol. Publicadas por el Licenciado Luis Tribaldos de Toledo, cronista mayor del Rey nuestro señor por las Indias, bibliotecario del conde de Olivares, Duque y gran canciller \&o. Dedicadas a Don Vicente Voguera, referendario de ambas signaturas de su Santidad, del Consejo de las dos Magestades, Cesarea y Catolica, gentilhombre de la cámara del Serenisimo Archiduque de Austria, Leopoldo. Con todas las licencias necesarias. Lisboa, por Pedro Craesbeeck, impresor del Rey nuestro señor, año 1625. A costa de Antonio Luis, Mercader.4 Bien que l'éditeur ne le dise pas formellement, la part que prit Noguera à la divulgation de ces œuvres oubliées d'un des meilleurs poètes lyriques castillans du XVI c sic̀cle ne se borna à la restitution du manuscrit qui lui avait été remis ni aux approbations ver-

1 „Alli [en la Suprema Suplicacion de los reinos de Portugal] se consumó, juntando á la grande téorica dellos [los derechos civil y canónico] grande práctica forense en seis años de continuo ejercicio judicativo." Dédicace des Obras de Francisco de Figueroa, Lisbonne 1625.

2 Le traité de Bento Gil, Directorium advocatorum et de privilegiis eorum, imprimé (d'après Barbosa Machado) sous les auspices de Noguera, à Lisbonne en 1613, renferme peut-être quelque chose sur les débuts de notre érudit dans la magistrature ou sur sa vie antérieure, mais je n'ai pas pu voir ce livre.

3 „Pasados los cuales [seis años],... tomó V. m. aquella resolucion tan controvertida de renunciar su plaza, y vacar solamente á las letras y estudios superiores, por serlo su genio, y se vino á esta córte á hacer dejacion della." Dédicace des Obras de Francisco de Figueroa.

+ Je ne connais de cette édition princeps que la description et les extraits dunnés dans l'Ensayo de una biblioteca española, t. II, col. 1071-1075. 
sifiées qu'il s'était chargé d'ohtınir de I.ope de Vega et de Juan de Jáuregui pour orner le livre dı poète d'Alcalá et en recommander la lecture: je montrorai plus bas qu'il aida aussi de ses deniers cette publication posthume. Dans sa dédicace, dont j'ai déjà eu l'occasion de tirer plusicurs renseignements, Tribaldos, après toutes sortes d'éloges, complète la liste des connaissances philologiques de notre portugais: „V. m. posee como maternas las lenguas hebrea, caldea, griega, latina, italiana, francesa, sin la nuestra natural, y el mas que mediano conocimiento de la siriaca, arábiga, tudesca, y el mediano de la turquesca, persiana y etiópica: de todas las cuales ha procurado saber con particular estudio los fundamentos y reglas, trayendo a gran costa maestros de muy distantes reinos, por conocer que quien esto no hiciese, no podria penetrar los secretos que en semejantes lenguas estan depositados". Voilà certes un homme bien pourvu et à qui il eût été facile d'appliquer ses connaissances linguistiques à l'élaboration de travaux sérieux dans le domaine de la philologie classique ou orientale. Pourquoi faut-il que cette science, acquise au prix de longues veilles et de grands sacrifices, soit restée stérile? Assurément ce ne furent pas les loisirs qui manquèrent à Noguera: tout porte à croire qu'il continua sa vie d'érudit, retiré du monde, jusqu'à son arrestation en 163I. Il est vrai que Tribaldos décore son protecteur, sur la couverture du recueil de Figueroa, des titres de référendaire des deux signatures de Sa Sainteté, de conseiller des Majestés Césarée et Catholique et de chambellan de l'archiduc Léopold, ce qui semblerait indiquer qu'il était rentré avant I625 dans la vie publique. Mais les charges de chambellan et de conseiller ont pu être purement honorifiques ', et quant à celle de référendaire des deux signatures, tout ce que je puis en dire c'est que la cour de Rome, en vertu d'une bulle de Sixte-Quint, exigeait des candidats à ces fonctions le titre de docteur utriusque juris, deux années de séjour à Rome, plus de vingt-cinq ans d’âge et une certaine tortune ${ }^{2}$. Noguera aurait-1l abandonné son musée pendant deux ans pour obtenir une charge médiocre, qui, après son retour au Portugal, ne pouvait lui rapporter aucun avantage pécuniaire? Cela est peu probable, et j'aimerais micux croire que les formalités prescrites par la chancellerie pontificale n'étaient pas strictement observées, et que Noguera obtint cette patente de référendaire à titre honorifique, sans quitter ses pénates.

Deux ans plus tard, en 1627 , lorsque le même Luis Tribaldos de Toledo mit au jour la première édition de la Guerra de Granada, par Diego Hurtado de Mendoza ${ }^{3}$, qu'il adressa cette fois

- Dans la dédicace de la Guerra de Granada Tribaldos parle d'une gráce concédée à Noguera par une cédule de Philippe III, du 3 octobre I620, mais il n'en indique pas l'objet.

2 Relatione della corte di Roma ... dal Sig. Cavalier Girolamo Lunadoro, Bracciano, I649, p. 79.

3 Guerra de Granada. Hecha par el Rei de España don Philippe II, nuestro señor, contra los Moriscos de aquel reino, sus rebeldes. Historia 
encore à Noguera, celui-ci vivait toujours à Lisbonne et jouissait des trois charges dont il a été fait mention. Cette publication de l'œuvre historique du célèbre écrivain grenadin est sans doute le meilleur service que Noguera ait rendu dans toute sa vie aux lettres nationales. Et l'on est autorisé à rapporter à cet érudit le principal mérite de l'entreprise, car, indépendamment des conseils qu'il prodiga à Tribaldos ', Noguera, je le ferai voir tout-à-l'heure, se chargea des frais ou d'une bonne partie des frais de l'édition princeps. Peut-être même fit-il quelque chose de plus pour honorer la mémoire de Diego de Mendoza. Soarez de Brito parle de notes marginales apposées par D. Vicente au texte de la Guerra de Granada, et bien que Tribaldos n'en fasse nulle mention dans les préliminaires de l'édition de 1627 , rien n'empêche qu'on ne les retrouve un jour ou l'autre dans un volume quelconque de papeles zarios. Je n'ai pas hésité à qualifier de princeps l'édition de la Gucra de Granada, publice à Iisbonne en 1627 , et à reconnaître ¿̀ Noguera et à Tribạldos l'honneur d'avoir enrichi la bibliographie espagnole d'une ceuvre de premier ordre, que des considérations politiques et personnelles n'avaient pas laissé mettre en circulation jusqu' alors: c'est qu'en effet l'existence d'une édition antérieure à celle de 1627 est absolument controuvée. Nicolas Antonio est le coupable, c'est lui qui a noté, par inadvertance, dans sa Bibliotheca noz'a, une édition de Madrid, I6 Io, de Luis Tribaldos, que persome n'a jamais vue ${ }^{2}$, et l'erreur commise par l'illustre savant a été soigneusement répétée jusqu'à nos jours ${ }^{3}$ et le sera sans doute encore, quoiqu'on dise et quoiqu'on fasse. Pourtant le problème est facile à résoudre. Tribaldos dans l'avis au lecteur de l'édition de 1627 (comme l'a déjà montré Vicente Salvá) expose que les vérités trop crues et les nombreuses allusions personnelles des mémoires de D. Diego en ont empêché pendant longtemps la publication, mais que désormais , le danger a cessé, car près de soixante ans se sont écoulés (depuis les évèvements) 4 et aucun de ceux dont l'histoire parle n'est vivant". Ainsi, au dire de l'éditeur,

escrita en quatro libros. Por don Diegro de Mendoģa, del consejo del Emperador don Carlos $V$, su embaxador en Roma $i$ Venecia, su governador $i$ capitan seneral en Tosiana. Publicada por el licenciado Luis Tribaldos de Toledo, ihronista mayor del Rey, nuestro señor, por las Indias, residente en la corte de Madrid, $i$ por el dedicada a don Vicente Noguera, referendario de ambas sisnaturas de su Sanitidad, del Consejo de las dos Magestades Cesarea $i$ Catholica, gentilhombre de la Camara del archiduque de Austria Leopoldo. Con todas las licencias necessarias. En Lisboa. Por Giraldo de la Viña. Con prizilegio. Año 1627.

1, ,Fucron sus exhortaciones $\mathrm{i}$ gusto el mayor motivo para que yo la publicasse", dit Tribaldos lui-même. (Dedic. de la Guerra de Granada).

2 ,,Certe haec historia in schedis Mss. diu cursitavit per omnium manus... demum in lucem prodiit Ludovici Tribaldi Regii chronographi opera, Matriti 1610 , in 4 . Deinde Olisipone apud Craesbek I627.“ Bibl. hisp. nova, t. I, p. 291.

$:$ Notamment dans l'édition. de Valence de $1776, p$. III, dans celle de la Biblioteca Riz'adeneyra (p. D. Cayetano Rosell), p. VIII, note I, et dans le Diccionario de Tomas Muñoz y Romero, p. 130.

- La guerre contre les Morisques commença, on le sait, en 1569 . 
ce n'est qu'en I627 qu'on a $p u$ songer sans crainte d l'impression de ia Guerra de Granada: plus tòt l'entreprise eût présenté de sérieux inconvénients, des dangers mîme, auxquels un historiographe officiel, un serviteur du (omte-I)uc tel que Tribaldos n'aurait certes pas voulu s'exposer. L'édition de í 6 io n'a donc jamais existé ${ }^{1}$ : tout ce qu'on pourrait admettre, si l'on tient absolument à conserver quelque valeur à la notice d'Antonio, c'est que Tribaldos prépara longuement l'impression du livre (à partir de I6ro peut-être) et qu'il attendit pour le publier d'avoir vu disparaître les derniers acteurs du dramé de Grenade et d'avoir trouvé un amateur disposé à prendre à sa charge les frais de l'édition.

A deux reprises déjà j’ai fait allusion à l'appui matériel que Noguera prêta aux travaux littéraires de l'érudit Tribaldos, me réservant d'en donner plus tard des preuves: c'est ce que je vais faire en introduisant le lecteur dans le musée du chanoine portugais, dont un inventaire sommaire, dressé par les soins du fisc, nous a fort heureusement conservé la mémoire. La Bibliothèque Nutionale de Paris possède dans sa collection de manuscrits portugais (no. 5I) un mince volume in-folio, relié en parchemin aux armes de Portugal, qui contient le relevé de tous les livres composant la bibliothèque de Noguera, au moment de son arrestation, en I631. Ce catalogue, intitulé „Rellaçaõ da livraria de Viçènte Nogueira confiscado", est distribué par matières en plusieurs chapitres, dont voici la liste: „Memoria dos livros pertencentes a Theologia“ (ff. I - 9 ); "Memoria dos livros pertencentes as faculdades de canones e leys" (ff. 9-I 8); „Memoria dos livros pertencentes ao spiritual" (ff. I $8^{\mathrm{vo}}-22$ ); "Memoria dos livros pertencentes a philosophia“ (ff. $22-25^{\text {vo }}$ ); "Memoria dos livros pertencentes a historia“ (ff. 26-47); "Memoria dos livros pertencentes a humanidades" (ff. $47-58^{\text {ro }}$ ); "Memoria dos livros pertencentes a Mathematica, Architectura e Musica" (ff. $58^{\circ 0}-66$ ); "Memoria dos livros pertencentes a Medicina“ (fi. 66-68); „Memorial dos livros pertoncentes a Politica" (ff. 68-70); „De: miliçia“ (f. 79); „Inventario particular dos livros defezos que se separaraó de toda a livraria do conego Vicente Noguera confiscado" (ff. 73-82), et ce dernier chapitre se termine par cette remarque de la personne chargée de procéder à la saisie: „listos livros defesos entraraõ como tais na Inquisiçaõ por ordem que para isso ouue". Iedit catalogue, quoiqu' assez mal rédigé, suffit à faire connaitre en gros les richesses bibliographique's cle Nogucra. L'érudit portugais possédait un bon fonds de bibliothéque, peu de grandes raretés, à peine quelques manuscrits, mais en revanche beaucoup d'excel-

1 M. William I. Knapp a observé en outre, dans le prologue des Obras poéticas de D. Diegro Hurtado de Mendoza, Madrid, 1877, p. XXIII, que l'édition de la Guerra de Granada de Madrid 1674 est qualifiée par l'imprineur de segrundo buelo, ce qui n'aurait pas eu lieu, si l'édition de 1627 n'avait pas été la première. J'ai moi-même ajouté quelque chose a ces arguments, vi:j. l'Espag'ne au XVle et au XV'lle sièile', p. 67\%. 
lents livres de travail, et si les circonstances s'y étaient prêtées, il serait arrivé sans aucun doute à réunir une librairie comparable aux collections des célèbres bibliophiles de son temps, tels que le connétable de Castille et Lorenzo Ramirez de Prado. Ses livres prohibés n'offrent pas, comme on pourrait le croire, un bien grand intérêt; on "cherche en vain dans cette liste les écrits des hétérodoxes espagnols du XVI ${ }^{\mathbf{e}}$ siècle, depuis les Erasmiens jusqu'aux Calvinistes, et l'on y trouve, par contre, des choses, fort inoffensives, des traités de Vives, les poèmes de Dracontius (!), des dissertations d'Antonio Agustin, Xénophon (!!), et généralement tous les livres écrits en grec ou dans une langue sémitique. Les inquisiteurs évidemment jugeaient plus simple de condamner tout ce qu'ils n'entendaient pas. C'est à la fin de la section historique (f. $46^{\circ 0}$ ) qu'on trouve la mention des ouvrages acquis en nombre par Noguera: „Dom Diogo de Mendoça guerras de Granada, tresentos sesenta e quatro volumes, a quarenta rs cada hum, emportaơ quatorze mil quinhentos sesenta rs, e todos estað em papel pera se encadernar"; - „Hispanica Historia, cento e vinte e tres volumes, a quatro rs cada hum, em papel pera se encadernar, valem quatro centos e noventa e dous rs"; - „Figueroas, tambem em papel, seiscentos e trinta e nove volumes, a oito rs cada hum, valem cinco mil cento e doze rs". - "Garcilassos noventa e seis volumes, tambem em papel, a oito rs, valem setecentos sesenta e oito rs". Diogo de Mendoça et Figueroas désignent clairement les éditions de Tribaldos: de l'une Noguera prit trois cent soixante quatre exemplaires, de l'autre six cent trente neuf, et ces chiffres ne représentent probablement pas la totalité de la souscription, car à l'époque de la saisie le Mécène portugais s'était sans doute déjà défait d'une partie de ses acquisitions. De toutes manières l'assistance pécuniaire de Noguera est bien établie et peut passer pour considérable, si l'on tient compte que le tirage de tels livres ne devait pas alors dépasser de beaucoup le chiffre de mille exemplaires. Quel est l'ouvrage désigné sous le titre d'Hispanica historia? Est-ce encore un travail commandité par Noguera, ou une production originale? I Quant au Garcilaso, dont Noguera prit d sa charge quatre-vingt-dix exemplaires, je pense qu'il s'agit de l'édition de ce poète, publiée à Madrid en 1622 par Tomas Tamayo de Vargas, savant très lié avec notre portugais et qui accepta son appui dans une autre occasion, comme on le verra plus bas par le Discours sur la langue et les auteurs d'Espagne. ${ }^{2}$ - Un autre article du catalogue mérite l'attention, il est ainsi conçu (f. 44):

\footnotetext{
1 Peut-être la Summa Historiae Lusitanae citée par Soarez de Brito.

2 Je dois dire cependant que D. Marcelino Menendez Pelayo, qui a bien voulu examiner pour moi cette édition de Tamayo, n'y a trouvé d'autre allusion à Noguera que ces mots-ci (p. 83): „De los versos castellanos (de Garcilaso), demas de los que havemos hallado, pudieramos añadir otros, devidos á la diligencia $i$ curiosidad de don Vicente Noguera i de don Francisco López de Aguilar, que con liberalidad me los comunicaron, si no temiera las diudas de nuestros Aristarchos."
} 
„Poesias de Dom Diogo de Mendonça emendadas pera se imprimirem, pergam., quatro centos rs “, c'est-it-dire recueil, préparé pour l'impression, des poésies de Diego de Mendoza. Or, on sait que les poésies du grand écrivain furent imprimées, moins les obras de burlas, à Madrid en 16 Io par lirey Juan Diaz Hidalgo. Noguera aurait-il acquis le manuscrit de cet éditeur, ou bien, mécontent du travail d'Hidalgo, aurait-il lui-mème mis la main à l'œuvre et réuni les matériaux d'une nouvelle édition? La note du catalogue ne permet pas de trancher la question: il faudrait retrouver le manuscrit. Avis encore aux érudits portugais.

„Le 17 juin [163I] je fus arrêté“. Voilà en quels termes, par trop laconiques, Noguera rend compte, dans son Discours adressé à Francesco Barberino, du grave évènement qui lui fit perdre la haute situation qu'il s'était créée au Portugal et motiva son exil, après un emprisonnement de plusieurs années peut-être. Noguera craignait-il de découvrir ì des étrangers le motif de son arrestation et de sa fuite de la Péninsule? C'est vraisemblable. En tous cas il évitait de se compromettre par écrit - scripta manent -, mais dans la conversation il se montrait évidemment moins réservé, et la preuve en est qu'un personnage considérable, le P. Dom Christophe Dupuy, procureur général de l'ordre des Chartreux, qui accueillit Noguera un certain temps après son arrivée à Rome, donne à entendre qu'il a été mis au courant de l'affaire. L'extrait suivant d'une lettre écrite par cet ecclésiastique, d. Rome, le 4 juin 1636, à son frère Jacques Dupuy à Paris 1, va nous initier aux débuts de l'exilé dans sa nouvelle résidence:

„Un Espagnol, agé de cinquante cinq ans ou environ, homme de lettres, fort versé es langues latine, greque, hebraique et arabique, a recerché il y a long temps occasion de me voir, a cause de l'amitié que il avoit eu aultrefois avec feu M. le president de Thou, de qui il savoit que j'estois parent. Il fut dans la semaine passée, et fusmes environ ensemble deux bonnes heures, pendant lesquelles il me raconta combien il estimoit et honoroit la memoire de $M$. le president de Thou et affectionnoit ceux qui lui toucherent comme nicy, que l'on lui avoit dit estre fils de Claudio Puteano; me dit que par l'entremise de M. le baron de Senecey ", ambassadeur en Espagne, il avoit entretenu correspondance par lettres avec le dit $\mathrm{Sr}$ President, de qui il avoit receu aussi de tres belles et longues lettres, et que il avoit eu occasion de le servir en Espagne sur plusieurs informations appartenant a son Histoire qu'il desiroit avoir de ce pays la, ou il estoit lors en quelque consideration; mais que a present pour quelques intrigues de court il avoit esté contraint de se retirer de son pays pour se tenir a couvert a Rome, ou il est conneu de fort peu de gens, se faisant appeller Dom Francisco della Noia, qui est un nom supposé, affin d'en estre par ce moyen moins reconnu, et me dit son vrai nom, dont je n'ai pas pour le present souvenance, sous lequel il a escrit a Mons. le President, et je croi que parmi les papiers nous pourrions encore treuver ces lettres. Il me souvient que $M$. de Thou me dit aultrefois que il avoit receu quelques lettres d'un Espagnol, home de savoir et de grande condition,

1 L'original de cette lettre se trouve dans la collection Dupuy, vol. 730 , f. 5 à 6.

2 Henri de Baufremont, marquis (et non baron) de Senecey, ambassadeur extraordinaire en Espagne, du mois de novembre 1615 au mois d'octobre 1618 . Ses dépéches se trouvent à la Bibliothèque Nationale, Collection Harlay, Finds Franç. 16116 et 16117. 
et qu'il s'estonnoit comment un home de ce pays escrivoit si bien et $2 \mathrm{Hec}$ tant de liberté. Au reste il conoist parfaittement tous les bons livres, et con il a une grande memoire, scait toutes les particularités de beaucoup de fact. les de France, est informé de l'amitié que mon pere avoit avec Mosr Pithoúu Mons. le Fevre ะ, M. de l'Escale ${ }^{3}$, dont il a admiré les œuvres, a veu quelques lettres que ce dernier nous a escrittes et se souvient de tous ceux qui ont fait mention de feu mon pere en leurs escrits. Je lui fis present d'un portrait de $M$. de Thou, que il contempla fort longtemps assez fixement, me demanda de quel pais il estoit, si ses enfans lui ressembloient de visage aussi bien que de valeur et entre aultres son aisné 4 , du merite duquel il estoit fort bien informé, ayant sceu mesme que il estoit destiné pour l'ambassade du levant, me remercia avec de grands complimens du portrait, et que il falloit que je fusse bien courtois, puisque a notre premiere veüe je l'honorois d'un si digne present. Je vous prie de recercher les lettres de notre home et de me mander, si vous les treuvez, ce que elles traittent. Je n'ai point reconneu en cette premiere entreveüe comment il est appliqué aux affaires du monde, mais je juge que il en a une grande conoissance, puisque il est si curieux, qu'il a beaucoup leu et qu'il a une grande memoire. L'on m'a dit que il parle des affaires presentes comme celui qui est hors de tout interest et ni temoigne aucune passion: cela se descouvrira puis apres par sa conversation, et vous donnerai avis de tout."

Quelques intrigues de cour: telle fut la cause du désastre. Le renseignement est un peu vague, mais il suffit pour enlever tout prétexte à une supposition qui involontairement se présentait à l'esprit, à savoir que Noguera aurait payé de sa liberté et de sa situation personnelles l'indépendance relative de ses opinions religieuses et philosophiques. Certaines phrases de sa lettre à de Thou et de son Discours, d'autres indices épars dans la correspondance de plusieurs savants étrangers, enfin l'intervention de l'Inquisition dans le séquestre de ses biens, tout cela donnait à penser qu'il n'avait pas dû s'en tenir toujours au credo espagnol de l'époque, et qu'une manifestation imprudente de quelque hérésie avait pu provoquer son arrestation. Maintenant, et tout en reconnaissant d'ailleurs à notre portugais un esprit assez libre et avancé pour son pays et son temps, il convient, devant le témoignage formel du P. Dupuy, de ne plus voir en lui qu'une victime de: quelque tripotage politique ou plus probablement de quelque rancune personnelle. Ce qui l'engagea ì se réfugier a Rome, après avoir fui l'ingrate parite, ce fut vraisemblablement l'assurance qu'il devait avoir d'y citre bien accueilli en sa qualité de référendaire des deux signatures et de chanoine de la cathédrale de Lisbonne; puis Rome était alors un centre scientifique de premier ordre, un asile sûr pour les érudits maltraités par la fortune, qui trouvaient là facilement à s'introduire dans le famulatus d'un dignitaire quelconque de la cour pontificale. Je ne saurais déterminer exactement l'époque de l'arrivée de Noguera ì Rome. Dupuy écrit au

1 Pierre Pithou, ou son frère, François Pithou, tous deux également érudits.

2 Nicolas Leferre, excellent humaniste, ami de Pierre Pithou.

3 Josephe - Jules Scaliger.

- François Auguste de Thou, conseiller du Roi et grand maître de sa bibliothèque, décapité le 12 septembre 1642 pour avoir pris part à la conjuration de Cinq-Mars. 
mois de juin 1636, comme on vient de le lire, que depuis longtemps l'exilé portugais insistait pour le voir, ce qui peut s'appliquer indifféremment à une période de plusieurs années ou de plusieurs mois; cependant comme aucun des documents qui nous renseignent sur le séjour de Noguera dans la ville éternelle ne remonte au dela de l'année 1636 , on peut admettre comme probable qu'il vint s'y établir vers cette époque. ${ }^{1}$

Très peu de temps après son installation, Noguera, ou, pour l'appeler par son nom de guerre, Francesco di Noya fut mis en rapport, par l'intermédiaire de Jean-Jacques Bouchard ${ }^{2}$, avec un des plus grands savants de l'époque, un des plus généreux amis des sciences et des lettres qui aient jamais existé, le célèbre Nicolas Claude Fabry de Peiresc. De son splendide musée d'Aix, où venaient s'amonceler ces innombrables trésors bibliographiques et archéologiques, dont les épaves font encore aujourd'hui notre admiration, Peiresc avait l'œil ouvert sur tout ce qui se produisait en Europe d'important ou de curieux aussi bien dans le domaine de la politique que dans le cercle de ses études favorites. Tenu, en particulier, très au courant des nouveautés de Rome par ses nombreux correspondants, il ne pouvait manquer d'être averti de l'incident Noguera, et une fois informé sur le mérite exceptionnel du sujet, ses antécédents d'homme de cour, de savant et de collectionneur, le conseiller d'Aix devait se sentir attiré vers ce personnage assez mystérieux, qui peut-être en savait long sur beaucoup d'affaires, ou en tous cas était bien préparé à répondre à ses questions sur les choses littéraires d'Espagne. Ce fut naturellement Noguera qui entama la correspondance. Sa première lettre ne nous est pas parvenue: cette lacune est fort regrettable, toutefois je ne pense pas que la missive contînt des détails autobiographiques très circonstanciés. En écrivant pour la première fois à un étranger inconnu, l'exilé a dû se tenir sur ses gardes et ne dire que juste ce qu'ii en fallait pour se rendre sympathique et intéressant. Pcircsc, de son côté, ne se compromit point et nc répondit qu'après avoir consulté Jacques Dupuy, à qui il demanda entre autres si ce que disait Noguera de ses rapports épistolaires avec de Thou était exact. Laissons-le parler ${ }^{3}$ :

„Un bonhomme Espagnol Portugais s'est retiré a Rome incogneu, qui a esté fort persecuté en son païs, lequel estoit de la cognoissance particuliere de feu Mr le president de Thou, et avoit belle bibliotheque et grande curiosité,

- Dans son Discours sur la langue et les auteurs d' Espagne, qui est daté du 5 janvier 1637, Noguera parle d'un ms. de l'Escurial, qu'il dit avoir examiné ,il y a environ vingt mois.“ Donc au printemps de 1635 notre homme était encore en Espagne.

${ }^{2}$ "Conciliarat hunc (comitem Fridericum Ubaldinum) illi (Fabricio de Peiresc) amicus Buccardus, ac simul Vincentium Nogueram Lusitanum nobilem." Viri illustris Nicolai Claudii Fabricii de Peiresc, senatoris Aquisextiensis, Vita. Authore Petro Gassendo, etc. (à l'année 1636), Hagae Comitum, $3^{e}$ éd. 1655, $4^{4}$, p. 196.

3 Lettre originale de Peiresc à Jacques Dupuy, prieur de S. Sauveur, Aix, 19 aout 1636. (Bibl. Nat. Collection Dupuy, vo!. 718, f. 289).

Zeitschr. f. rom. $\mathbf{P h}$. III. 
mais il est incognito dans Rome soubs un autre nom que le sien et ne desire pas d'estre descouvert. Il m'a escript la lettre que vous pourrez voir, à laquelle je ne feray pas de responce que je n'en aye vostre advis, et serai bien aise de sçavoir si vous avez poinct rencontré de ses lettres parmy les papiers de feu Mr de Thou et si l'escritture en est pareille ou non. C'est homme pourroit bien sçavoir des intrigues et en descouvrir si l'on vouloit. Vous le mesnagerez selon votre bon plaisir au cas que vous trouvassiez à propos d'en parler. Puis que feu $\mathrm{Mr}^{\mathrm{r}}$ de Thou n'avoit pas desdaigné sa correspondance, il pouvoit bien avoir des qualitez dignes de n'estre pas negligées."

Quelques jours plus tard, après avoir reçu une réponse de Dupuy, Peiresc revint à la charge $\mathbf{1}$ :

"J'ai grande compassion de ce pauvre Portugais, puisqu'il est de la qualité portée dans les lettres de Mr Holstenius² et comparable a de si grands hommes de lettres, le voyant si mal traicté comme dict le R. P. D. du Puy. L'envie et la jalousie de l'ignorance produict tousiours de ces effects contre les plus vertueus. J'avoys creu à l'ouyr parler que la correspondance eust esté bien plus grande avec feu $\mathrm{Mr}$ de Thou et de plus longue main, mais tousiours est ce prou d'avoir mérité une responce de la part d'un si grand personnage. Si nos cartes n'estoient si brouillées, possible y auroit-il moyen d'en tirer quelque advantage com' avoit faict autres foys le feu roy d'Antonio Perez, et possible est ce à quoy il en vouldroit bien pouvoir venir, s'il y avoit quelque disposition."

Je ne puis rien dire, malheureusement, des lettres de Peiresc à Noguera, ne les ayant point retrouvées. Il y en a eu cependant. Noguera affirme qu'il en a reçu, au moins une ${ }^{3}$, et leanJacques Bouchard mentionne expressément l'érudit portugais parmi les correspondants de son ami ${ }^{4}$, mais ni nos manuscrits de Paris ni les recueils des minutes de Peiresc déposés à la bibliothèque de Carpentras n'ont conservé aucune copie de cette correspondance ${ }^{5}$; quant aux originaux, qui ont dú séjourner un certain temps à Rome, sait-on ce qui en est advenu!

C'est à la fin de l'année 1636 que le conseiller d'Aix demanda à son "bonhomme" une consultation sur la littérature espagnole (castillane et catalane); il ne s'adressa pas à lui directement, mais au cardinal Francesco Barberino, neveu du pape Urbain VIII, sous l'égide duquel Noguera vivait alors a Rome en compagnie de bien d'autres savants ${ }^{\circ}$. Peiresc, pour mieux servir

1 Lettre originale de Peiresc au même, Aix, 9 septembre I636 (Collection Dupuy, vol. 718, f. 299).

$\because$ Voy. plus bas un passage d'une lettre de Holstenius relatif à Nogucra.

3 Voy. plus bas sa lettre à Peiresc.

4 ,Extant (epistolac Peireskii) de literis, quas humaniores vocant, linguisque tam veteribus, Europacis, Asiaticis, atque Africanis, quam recentioribus, Gothica, Cantabrica, Provinciali, atque Italica; ad Lucan Iolstenium, Gabrielem Naudaeum, Ieonem Allatium, Vincentium Nogrueram, \& Federicum Ubaldinum; viros in iis summos atque perfectos." Peireskii laudatio habita in concione funebri Academicorum Romanorum, die decembr. 21 an. 1637 , Fo. Facobo Buccardo Parisino perorante (dans la vie de Peiresc par Gassendi, éd. citéc, p. 257).

5 M. Tamizey de Larroque, qui possède comme personne son XVIIe siècle et qui, en particulier, sait sur Peiresc et ses amis tout ce qu'il est possible de savoir, a bien voulu examiner, sur ma demande, les copies qu'il a prises des recueils de Carpentras: il n'y a rien trouvé qui fasse allusion a Noguera. 'Les relations de Noguera et du cardinal Francesco Barberino datent 
son correspondant, eut l'idée délicate de faire présenter au cardinal le travail qu'il avait demandé pour lui-même. Ce mémoire, dıont l'original fut remis à Barberino et dont une copie, exécutée par Holstenius, fut envoyée à Peiresc, n'est autre chose que le Discours sur la langue et les auteurs d'Espagne, que nous imprimons plus bas d'après cette même copie du savant de Hambourg.1 Quelques jours après avoir terminé et présenté son mémoire, Noguera rendit compte à Peiresc de ce qu'il avait fait pour répondre à ses désirs. Sa lettre, datée du 27 janvier 1637, contient divers détails curieux, elle a de plus pour nous un prix tout particulier, car c'est le seul document autographe que nous possédions de notre auteur. La voici: ${ }^{2}$

„Illmo e $\mathrm{R}^{\mathrm{m} n}$ Signore, Pation mio cclmo, Mancano senipre le parole in occorrenze di grande maraviglia e non sperate, e quantumque dalla benignità di V. S. Ina mi promettevo ogni favore e buona corrispondenza, non pur l'aspettava in grado si eccessivo, donde vengo a giudicar per diffettuosi quanti ho sentito parlar de V. S. I., e non perciò gli incolpo, perche vince V.S. I. colle sue virtù tutte le hyperbole; e per non toccar verità che la modestia di V. S. I. sentirà malagevolmente, passo a renderli gratie infinite della diligenza fatta nel Mercurio francese, que quandocumque arriverà, sarà il benvenuto, e come vengano intieri, daro per ben impiegata ogni dilatione. Lessi gia tutti fin all' anno de 1631 , e quantunque la narrativa e stilo sia ordinario e manco che ordinario, pur insere bellissimi pezzi originali che rendono il tutto desiderabile. Supplicarei a V.S. I. non suspendesse le diligenze di cercarlo, senon sapessi esser superfluo appresso di lei ogni mio simile ricordo.

Il sre Cardle Patron mando al conte Ubaldini mi communicasse tutta la lettera che V.S. I. scrisse a S. Emza, ma il conte, o non intese bene il precetto, o come grande cortegiano volse limitarlo, o bene per giudicar che fuor de' quesiti di Spagna io mi sbaglarei, di soli questi mi diede ragguaglio, rogandome che io facesse questo presente al sre Cardinale, accioche da S. Emza $\mathrm{e}$ non da me V.S. I. il ricevesse; et io leggendo la lettera scrittami da V. S. I., trovai che anche V. S. I. per quella via voleva che io obligessi e servissi al sre Cardle, come feci presentandoli et anche legendoli la cartuccia. S. Emza si contentò di essa assai e mi disse encomii, perche attese più il animo dil iscrivente che la scrittura, e si stizzd tanto della cautela dil Conte, che gli mandò subito mi mostrasse tutti i quesiti di V. S. I.; ma una colica che nell' istessa hora affisse al Conte ha differito il far questo con util mio, che ciaciarando tanto nelle cose patie forsun obmutescerem aut sane balbutirem nelle externe: in quelle di proposito mi distesi, perche so che il genio di V.S. I. non si satisfa di notitie infarinate, ma di quelle che penetrano fin al centro, e che vorrebe in ogni materia csser informato fin delle piu minute circonstanze.

Non ho a mano La Noblezza di Andaluzia ${ }^{3}$, ma quando la piglierò dalla bibliotheca dil Sigre Cardle, rivederò la lista de' manuscritti e forse dirò de alcuni, pur V.S. I. in ciò che non saprà altronde sospenda il assenso, perche

peut-être d'Espagne; ce dernier vint en effet à la cour de Philippe IV, chargé d'une mission extraordinaire, en 1626. Voy. la Verdadera relacion de la entrada que hizo en esta corte Su Illustrma del señor Cardenal Francisco Barberino, sobrino de Su Santidad Urbano VIII y su legado a latere. Madrid 1626. (Copie à la Bibl. Nat. Collection Brienne, t. 55, f. 30-33.)

1 L'authenticité de la copie conservée dans la collection Dupuy est établie par la comparaison de lettres autographes de Holstenius qui se trouvent dans le ms. de la Bibliothèque Nationale, Nouv. acq. lat. 1212.

2 Bibl. Nat. Recueil de lettres adressées à Peiresc. Fonds Franç. 9540, f. 176.

3 Le nobiliaire bien connu de Gonzalo Argote de Molina, publié à Séville ep 1588 . 
mene accorgo che è gia la mia memoria molto debole e senile, e perciò non e cerimonia o modestia la protestatione che messi nelle ultime righe.

Trovai hoggi tra gli hibri di Alessandro Tassoni un Ausias March soprascritto da man sua, della 2 a editione, con un glossario de più di 90 anni. 1 Mandolo a V.S. I. per mano dil Sigre Cardle, perche è menor inconviniente trovarsi in essa bibliotheca duplicato che mancar afatto. L'istesso farei de gli altri Limosini, se qui si trovassero. Dissemi un erudito che il Tassoni nel principio delle sue Considerationi sopra il Petrarca, trattando de i Provenzali che gli precessero e degli cui studii lui se giovò, rifiuta un certo spagnuolo Villalobos \#, il quale afferma che il Petrarcha piglio da Ausias March, comettendo in questo un grandissimo anachronismo, forse di anni 100, havendo costui vissuto sotto il Papa Calixto $3^{0}$ e quello sotto gli Papi di Avignon. Non viddi ne il Tassone ne lo Spagnuolo e cosi non posso giudicarlo, ma solamente maravigliarme di tal sciochezza secundum ea quae proponerentur.

Il Sigre Cardle mi disse il suo pensiero intorno a stampar i versi e prose di quelli scrittori toscani che fiorirono col Boccaccio, cioe Franco Sacchetti, Guiton de Arezzo, Francesco Barberino, Cyno da Pistoia, che era far scelta de gli piu galanti, ma che V. S. I. era de parer che si stampasse tutto, e quale delle due opinioni mi piaceva più? Io gli dissi alla libera che quella di V.S.I., perche de artefici primarii anche gli scherzi e ciò che fanno senza cura merita riguardarsi, e cosi che S. E. nil istius modi nobis invideret.

Tres gratie domandai a N. S. ${ }^{3}$ e tutte tre mi fece per merito et intercessione dil Sigre Cardle, ma è tale la bassessa della mia fortuna che cio che ho avanzato con esse de restar nel stato copmune de gli altri huomini, perche senza esse stava nel più vile de tutti; pur me le fece con una conditione assai rigorosa, cioè dummodo in Italia remaneat. Dalla quale ho supplicato e spero moderatione, perche quantumque io non ho pensiero di uscir da essa, ne forsi da Roma, mi fa ascriptitium et veluti servum glebae e mi leva quella commune libertà colla quale potrò andar da V. S. I. e servirlo di capellano. Hoggidi et allora non potrò. $\mathrm{E}$ ben conosce il Patron che se $\mathrm{mi}$ fa alquanto di torto, ma è grande la potenza con la cui ombra si consultano qui tutte le risolutioni: utcumque res cadat mi trovarà disposto $\mathrm{e}$ paciente.

Se V.S. I. si degnera di favorirmi di lettere sue, lo havrò a gran honore, e si gli aggiunerà de suoi commandamenti, cum fove de felicitate contendam; perche stimo più la amicitia de V. S. I. che forse tutte, havendo per fondamento di parte de V.S. I. una maravigliosa doctrina e prodigiosa eruditione,

1 Noguera fait allusion à la seconde édition du texte catalan des poésies d'Ausias (ou mieux Auzias) March, publiée à Barcelone en 1545, laquelle contient "totes les declarasions dels vocables scurs molt largament en la taula." Voy. la description de ce livre dans Pedro Salvá, Catálogo de la biblioteca de Salvd, Valence, 1872 , No. 768.

2 Non pas Villalobos, qui n'a rien à faire ici, mais Juan Lopez de Hoyos, le précepteur de Cervantes. Voici du reste le passage de Tassoni: „Odoardo Gomez di Portugallo e Giacopoantonio Buoni Ferrarese scrissero che'l Petrarca, non da Toscani antichi, ne da Provenzali, ma da Ausias March, poeta lemosino, gran parte delle sue composizioni avea tolto.... Alle male accozzate testimonianze del Gomez e del Buoni, s'atterza quella del Maestro Juan Lopez de Hoyos, il quale nell' approbazione, che fé delle Rime di quel poeta tradotte in castigliano, di giudice fatto procuratore, volle aggiugnere anch' egli all' imprimatur questo miccino di coda: Por mandado de V. A. he visto este libro de poesia del famoso poeta Ausias March, el qual es poeta español, y escrivio en lengua lemosina, que es lengua entre catalana y valenciana, $o$, por mejor dezir, un misto de catalana y algo de gallega y valenciana. Está traduzido en castellano por Iorge de Montemayor. En lo que toca á sus conceptos es tan subido que los de muy delicado juizio creen que Petrarca tomó muchos, de los mas delicados que tiene, deste Autor" etc. Considerazioni sopra le rime del Petrarca d'Alessandro Tassoni. Col confronto de' luoghi de' Poeti antichi di varic lingue. Modona $1609,{ }^{80}$, f. prél. 3 .

3 Le pape Urbain VIII. 
congionta con esser il Mecenate di questa età, e dalla mia una somma observanza alle sue virtu et un obligo nuovo alla sua liberalità, e pur gli baccio le mani. Roma, 27 di gennaro 1637 . Continui V. S. I. sempre col nome di Francesco di Noyia, mentre non mi levo la maschera.

\section{Di V. S. Ima e Rma \\ aff mo et obligmo servitore}

Don Vicente Noguera.

Holstenius ne mit pas beaucoup d'empressement à recopier le mémoire de Noguera. Le 5 mars 1637 Barberino écrivait à Peiresc: „De i Poeti Spagnoli ne starò trattando con questo gentilhuomo Portughese, che ne hà scritto à V. S.“ 1 . Et ce ne fut qu'un mois plus tard, le 4 avril, que le cardinal put annoncer à son correspondant le départ de la copie: "Con li seguenti (corrieri) V. S. hara ricevuto la copia della lettera che tratta di Poeti Spagnuoli, non s'essendo potuta copiare in tempo" 2. Holstenius ne manqua pas, comme bien l'on pense, de s'excuser auprès de Peiresc; en même temps il lui fit un bel éloge de notre D. Vicente, qu'il n'hésita point à comparer aux plus grands humanistes d'Espagne ${ }^{3}$ :

„Epistolicam dissertationem de lingua veteri Hispanorum, et de praecipuis ejus linguae scriptoribus, dn. Vincentii Noguerae, cum mea manu describere rogerer, superiori cursore mittere non potui; tu pro tua humanitate moram excusabis. Aucturcm tibi notum esse existimo, virum sane humanissimum et qui cum praecipui nobilitate summam eruditionem conjunxit, et quidem talem ut Hispania aequis oculis animisque tantum splendorem ferre non potuerit: judicium homini summum et exquisitissimum, omnium linguarum peritia accurata ad miraculum usque, ut Moralibus, Resendiis, Nunnesiis, Pintianis, Vivis, et praecipuis ejus gentis hominibus accenscri jure possit. Si quid praeterea hujus g literás compelles, cum tuam amicitiam anxie affectet: haud sine fructu inter vos commercium erit."

Ce n'était pas la première fois que l'érudit allemand honorait ainsi son collègue. L'année d'avant il l'avait déjà chaudement recommandé à Peiresc, en insistant sur son érudition solide, sa critique exercée, et en le louant spirituellement d'avoir su se tenir en garde contre les honteuses et ridicules supercheries des plombs de Grenade et des chroniques de Flavius Dexter et de Julien de Tolede 4 :

"Sed quid nos inter mortuos haeremus diutius? Vivos tibi commemorabo viros aliquot doctos, quibus nunc in Urbe fruimur. Degit. Degit hic in Urbe Vinc. Noguera, nobilis Lusitanus, vir summe doctus Hebraeas, Graecas et Latinas literas, Pinciano, Suritae, Vivae, Ciacconio, Nunnesio comparandus. Nihil vidi humanius, nihil suavius hoc homine; unde non mirer male ipsi convenire cum superciliosa natione. Ridemus subinde ineptias gentis illius, quae Flavios Dextros et Julianos Toletanos, sinistro auspicio et plumbeo

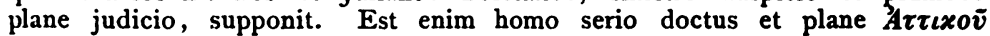
$\mu v \times \tau \tilde{\eta} \rho \circ$ s, cardinali nostro longe gratissimus."

L'année I64I nous apporte une épitre dédicatoire, d'un style des plus dithyrambiques, adressée à Noguera par le fameux $\mathrm{Za}$ -

1 Lettre originale de Barberino à Peiresc. Fonds Franç. 9537, No. 99.

2 Lettre originale du même au même. Fonds Franç. 9537, No. 95.

3 Lettre a Peiresc, de Rome, le 7 mars 1637. Lucae Holstenii epistolae, éd. Boissonade, p. 297.

1 Lettre au même, de Rome, 2 mai 1636; éd. citée, p. 499. 
cuto Lusitano, médecin d'origine juive (trop mal converti pour pouvoir vivre en paix dans sa patrie), qui vint se fixer à Amsterdam, où il entreprit la publication de ses deux ouvrages, le De medicorum principum historia et la Praxis historiarum. Les éloges de Zacuto, il faut le dire, n'étaient pas tout-à-fait désintéressés. Hoc mihi si dederis beneficium, quidquid splendidum, quidquid venustum, quicquid denique gloriosum mihi ex hoc opere continget, id omne tui nominis splendori et praeclarae stirpi tuae, nostrâ Lusitaniâ celeberrimae, inclytisque stemmatis illustri, assignabo: c'est en ces termes que le médecin portugais demande à son compatriote de délier les cordons de sa bourse ou peut-être simplement d'obtenir un secours d'un de ses patrons romains; et l'on peut supposer, sans malice, que le style de la pétition n'a pas dû déplaire à Noguera, pas plus qu'au cardinal, dont Zacuto chante les louanges en même temps que celles de son ancien ami. Au reste cette dédicace du tome VII de la Praxis historiarum n'enrichit pas de détails bien nouveaux la biographie de Noguera: on y apprend seulement que ce dernier trouva à Rome, outre Barberino, un autre protecteur également puissant, le cardinal Jules Saccheti, dont il avait peutêtre déjà fait la connaissance en Espagne pendant sa nonciature. ${ }^{1}$ Quoi qu'il en soit, il vaut la peine de reproduire au moins quelques passages de ce document. Le lecteur remarquera que la disposition typographique de l'adresse est à elle seule une flatterie:

Reverendo, illustri \& generoso domino, Divo Vincentio Nogueira, Quondam

Haereditario domino in Rios frios,

Olysipponensis Metropoleos Canonico,

Summi Pontificis, utriusque signaturae referendario,

Majestatum Caesareae \& Catholicae consiliario,

Leopoldi Austriae Archiducis clavis aureae cubiculario;

In privata fortuna

Nunc vero

Imo fortunarum omnium privatione,

Nulla tamen virtutis, doctrinae, famaeque minutione

Amico suo singularissimo Romae degenti,

Doctor Zacutus Lusitanus S. P. D.

Cum ex virtutis admiratione quasi bilance apprehensas doctissimorum hominum conditiones contemplor, cum collationem inter clarissima nostro sacculo coruscantia sidera tentare propono, cum eorundem ingenii \& dignitatis gradum adumbrare statuo, nonne par aut majus quid molior, ac si Anthaeum intra ulnas opprimerem, columnas in Oceano figerem, vel aurea Hesperidum mala raperem? Ex is occurris tu, eruditissime Vincentie, re \& nomine vincens peritissimos ... Quis enim Ciceronianam linguam, ejus flores, phaleras $\&$ calamistros nostro saeculo te elegantius colit, callet \& ornat? Quis Graecas literas, Chaldaicas, Syriacas, Arabicas intimius penetrat? Quis linguam sanc-

1 Saccheti séjourna en Espagne de 1624 à 1626; voy. Vicente de la Fuente, Historia eclesiástica de España, ze éd., t. VI, p. 449. L'année 1626 il fut élevé au cardinalat par Urbain VIII, et, plus tard, nommé préfet de la signature de justice: en cette qualité il devait avoir de fréquents rapports avec Noguera, référendaire des deux signatures. 
tam \& in ea recondita mysteria, ejus strenuos interpres acriori mentis acie, acutiori minervâ, maturiori consilio explanat faelicius in Europa tota? adeo, ut Rabbinorum peritissimi, te praesentem, divinaque elogia ex suavissimo ore exponentem admirentur, succenseant et contremiscant...1 Quo non possum non laudare \& summis usque ad astra evehere praeconiis Amplissimum Cardinalem Saccheti, universi orbis Atlantem fortissimum, qui te virum tantum, extra propriam patriam constitutum, in domum suam clarissimam recepit, fovit \& honoravit . . Tu certe, vir prudens, ingenuus, pius, bonus, doctus, bonarum literarum amantissinus, tam spectatissimi Principis favore \& dignitate stipatus, aliorum labores tibi oblatos in tuum securissimum umbraculum quasi tuo jure recipere teneris modo, sicut olim doctissima a celeberrimis viris tibi consecrata monumenta in tuam gratiam recepisti, auctoritate ornasti \& praemiis condecorasti. Hoc mihi si dederis beneficium, quidquid splendidum, quilquid venustum, quicquid denique gloriosum mihi ex hoc opere continget, id omne tui nominis splendori \& praeclarae stirpi tuae, nostrâ Lusitaniâ celeberrimae, inclytisque stemmatis illustri, assignabo. Ego enim amicitiam tuam ferventissime affecto \& sub cujus imperio vere desidero, non quia Lusitanus, \& a patria ipsa tua Olysippone oriundus, in qua ad summum fortunae apicem olim te erectum vidi, tot dignitatibus, muneribus splendidissimis cumulatissimum, justitiae presidem, consilio regio supremo assistentem circunspexi, sed ut beneficio tuo non difficulter in literarum palaestra honores maximos comparare queam. Vale ergo nostri gloria saecli, Hebraicae linguae coryphaeus, anterignanus \& antistes summus, \& me ex corde toto redama, ego enim in penitiori cordis mei sinu amorem tuum perennem alo, teque colo, veneror, observareque haud desinam durante vita. Amstelodami, 30 augusti, anno 1641. Anicitiae tuae firmissimae addictissimus,

Zacutus Lusitanus .M. D.2

Plus on avance dans la vie de Noguera, plus les documents se font rares ou, tout au moins, se dérobent aux recherches; et cependant la correspondance que l'émigré portugais entretint depuis son arrivée à Rome avec divers savants italiens ${ }^{3}$ et français n'a pas pu cesser brusquement: il avait trop d'intérêt à conserver

1 Ce dernier éloge, qui a déjà une certaine portée dans la bouche de Zacuto, est confirmé par l'illustre rabbiniste Jules Bartolocci. Traitant de la dédicace de Zacuto à Noguera, il dit: „In hac Epistola illum vocat Hebraicae linguae Coryphaeum et Antesignanum. Et revera fuit Vincentius Nougeira in Hebraica lingua admodum versatus. Vixit aliquandiu Romae in Palatio Cancell. Apostol. sub munificentia Card. Francisci Barberini. Obiit ibidem anno 1654, aetatis suae 68." Bibliotheca magna rabbinica Bartolocii. Pars II, Rome, 1678, p. 809.

2 Zacuti Lusitani, Medici \& Philosophi Praestantissimi Praxis historiurum. Ex limpidissimis solum principum fontibus exhausta ... Liber septimus. Amsterdam, 164I, in $8^{0}$, p. 3.

3 Noguera entra de bonne heure en relations avec le philosophe Fortunio Liceto, qui lui dédia le premier volume d'un de ses ouvrages, les De Quaesitis per epistolas a claris viris responsa Fortunii Liceti Genuensis, Bologne, $\mathrm{I} 640$, in $4^{\circ}$. In est même certain que notre portugais alla visiter le professeur italien, installé à Bologne à partir de l'année 1637 (voy. le P. Niceron, Mémoires pour servir a l'histoire des hommes illustres, t. XXVII, p. 376), car Holstenius termine une lettre adressée à Liceto, de Rome, le 2 avril 1638, $^{2}$ en disant: „Nobilissimum et eruditissimum Vincentium Nogheram peramanter a me saluta." Holstenii epistolae, éd. Boissonade, p. 300. Gabriel Naudé aussi, dans une lettre adressée au même Liceto, le 26 novembre 1638, fait saluer Noguera: „anlicosque supra morem aulae, singulari judicio praestantes multumque literis humanioribus excultos Vincentium Nogheram et Donatum Lintellum ... saluta." Gabrielis Naudaei... epistolae. Genève, 1667, in 120, p. 54 I. 
et à étendre ses relations scientifiques pour rompre un commerce épistolaire qui le posait très bien dans les cercles des lettrés romains; puis ses dernières années s'écoulèrent fort paisiblement, aucun accident fâcheux ne vint les troubler et il sut conserver jusqu'à la fin la faveur de son grand protecteur le cardinal Barberino, dans le palais duquel il mourut, au dire de Barbosa Machado. Cette pénurie d'informations doit donc être attribuée au hasard. D'ailleurs, je l'ai dit, je n'entreprends pas ici une biographie de Noguera: je ne fais que rassembler des notes qui me sont tombées sous la main, d'autres plus experts et mieux informés me complèteront et me corrigeront. En attendant voici encore quelques bribes, qui pourront servir au futur biographe. Le 3 mars 1644 le P. Christophe Dupuy écrit de Rome à son frère Jacques ${ }^{1}$ :

„Le Seigr D. Vincenzo Noghera m'envoye hier un livre que l'on lui a presté, intitulé $D u$ rappel des Fuifs, sans le nom de l'auteur ni du lieu ou il est imprimé, seulement l'année de l'impression y eșt marquée, savoir 1643 . Le livre est in $8^{0}$. Il n'est pas que vous ne l'ayez veu ou pour le moins que vous n'en ayez oui parler. Le seigr Vincenzo desire savoir l'auteur et l'estime que l'on fait de son ouvrage par dela, il m'en escrit en ces mots: „Io l'ho letto attentissimamente et ne sono restato maravigliato piu che di quanti libri ho letto da molto tempo in qua, perche l'autore fondandosi solo in luoghi delle scritture, intese a suo modo, discorre stravagantemente, ma con gran logica e gran probabilità, se gia non arriva a demonstratione, e con tanta pietà che tira molto il cuore all' amore di Dio e a non doversi villaneggiare li miseri Hebrei nostri (velimus nolimus) fratelli maggiori. Nel 20 libro va un poco stiracchiando la dottrina al $\mathrm{Re}$, che se intende del defonto, gia si sara accorto della mala congiettura, se del presente, sara piu improprio." Vous m'obligerez de me mander ce que vous aures apris de ce livre. J'ai eu quelque opinion que $M$. Gaffarel en pouvoit estre autheur, s'estant fort appliqué à ces doctrines de cabale."

Le I $^{\text {er }}$ mai suivant Dupuy revient sur le même sujet ${ }^{2}$ :

"J'ai fait voir l'endroit de vostre penultieme lettre, ou vous parlez de l'auteur $D u$ rappel, au Seigr Vincenzo Noguera, qui me dit aussitost que ce nom de Perere ${ }^{3}$ estoit espagnol, et pouvoit estre encore que celui qui le porte fust descendu de quelque Espagnol Juif comme il i en a quantité en Gascogne et en Languedoc. Je n'ai pas eu le loisir de lire tout ce livre a cause qu'il l'a fallu rendre ou Seigr Cav. del Porto, à qui il appartient, pour le faire voir a $M$. le Card. Barberino, qui en avoit une grande envie.“

Six ans plus tard Noguera reçut une assez longue lettre d'un de ses compatriotes, non moins célèbre par ses malheurs que par son talent littéraire, Francisco Manuel de Mello, l'auteur de l'Historia de los movimientos y separacion de Cataluña. Le prisonnier de Lisbonne prie son compagnon d'infortunes de faire publier à Rome un manifeste politique qu'il avait écrit dans sa prison; en même temps il s'offre à lui servir de correspondant politique et littéraire.

1 Collection Dupuy, vol. 730, f. 144 .

2 Collection Dupuy, vol. 730 , f. 155 .

3 Il s'agit ici du célèbre préadamite Isaac de la Peyrère, né à Bordeaux en 1594 (voy. l'excellente étude de M. Ph. Tamizey de Larroque, Quelques lettres inédites d'Isaac de la Peyrère à Boulliau, Bordeaux 1878, in 8\%). Si le nom de l'auteur du Rappel des fuifs était Peyrère tout court, la conjecture de Noguera aurait un certain poids, mais la présence de l'article rend cette prétendue origine espagnole fort douteuse. 
Plusieurs passages de cette lettre montrent que Noguera, après vingt ans d'exil, continuait à s'intéresser aux choses de son pays:

A Dom Vincente Nogueira, Referendario Apostolico de ambas a Signaturas, estando em Roma. ${ }^{\prime}$

Desde que à minha må chegou, pellas de N., hum capitulo de huma carta de V. M. escrita à N. (sobre aquella Historia dos Tavoras impressa em França, em a qual naõ tive pouca parte) 2 fiz proposito de buscar a V. M., por aquelle modo que a nossa distancia e a minha impossibilidade concediað. Varios accidentes me perturbàrao de sorte, que nem ainda agora, animado pello P. Mestre Fr. N., de todo me atrevera a offerecerme diante de V. M. com estas regras, porque verdadeiramente o desengano que de my tenho me faz ser encolhido. Conheço naz menos quantas razoẽs ha em V.M. paraque me queira contar no grande numero de seus affeiçoados; e julgo jà como por de maes a lembrança, que pudera fazer a V. M., das honras, com que sempre tratou de palaura e escrito a esse mal logrado ingenhe de D.N. meu tio. As que eu recebi iguaes em Madrid en este Reyno do Senhor N. E. julgo desta maneira; porque sey muito bem que com os grandes sogeitos serve de recomendaçaó a inorancia daquelles que os buscaő, a fim de se verem doutrinados. Para eu dar a V. M. inteira informaçað de minha fortuna, remeto a copia de hum Manifesto ${ }^{3}$, que fiz ha poucos tempos, de cujo credito resultou ser passado de outra prizaó a este Castello de Lixboa a onde fico, e donde com mayor comodo poderei empregarme em servir a V. M. no que por estas partes se oferecesse. Para informar a V. M. desse pequeno talento, que Deus foy servido repartirme, ouzo a offerecer a V. M. esses seis livros, parte dos que tenho publicado, e que bem poderaz servir de desengano ao pouco que de my ha que esperar. Alguns faltad dós impressos, porque os perdi pello mundo, emque andey perdido muitos anos, mas, para o que elles saž, muitos saź estes. Do mais dirà a V. M. ò P. Mestre Fr. N. Com tudo nao deixo de me louvar em S. Paternidade com escrupulo; porque segundo aqui foi servido de aprovar os meus nadas, receo que passe atè Roma com esta opiniaz. Bem me consta da universal curiosidade de V. M. e por mais que conheço terà V. M. neste Reyno grandes e sabios correspondentes, toda via eu me offereço a esse exercicio, se valer para tanto. Suprirà bem a industria a falta da suficiencia. Tenho aqui meus confessados dos negocios da Corte, dos quaes poderei dar a V. M. razaర como bem instruido. Tornando ao Manifesto eu dezejei naశ sem cauza es tampallo neste Reyno, a que fui persuadido dos grandes e dos grandes ministros. Respeito ouve para suspendello. Tivera hoje muito contentamento de que nessa Cidade se imprimisse na forma conveniente; para o que, logo que V. M. se servir de avizarme $u$ maudarà pur enı ubıa, eu achdiiey com os efeitos necessarios à despeza da officina por via do Padre Mestre Fr. N. ou pella que mais prontamente chegar a maós de V.M. A minha sede das boas

1 Primeira parte das cartas familiares de D. Francisco Manuel escritas a varias pessoas sobre assuntos diversos, recolhidas e publicadas em cinco centurias por Antonio Luis de Azevedo ... Roma, Na officina de Filipe Maria Mancini. MDCLXIV, in $4^{0}$, p. 235.

" Historia dos varnens illustres do apellido Tavora, continuada em os senhores da caza e morgado de Caparica... Recolhido pellas memorias originaes de seus passados, por Alvaro Pirez de Tavora, senhor da dita casa ... e publicado por Ruy Lorenço de Tavora. Paris, Cramoisy, 1648, in fol.

3 Dans sa liste des œuvres de Mello, Innocencio Francisco da Silva cite un Manifiesto de Portugal, imprimé à Lisbonne par Paulo Craesbeeck en 1647, et qui, dit-il ,tinha por fim patentear ao mundo a detestavel acção commettida pelo governo de Hepanha, quando por desfazer-se d' elrei D. João IV, o mandára assassinar atraiçoadamente no acto da proçisșăo de Corpus Christi em 17 de junho do referido anno." Mais Mello parle ici, en 1650, d'un travail récent et déclare en outre que certaines considérations en ont empéché la publication au Portugal: ce manifeste n'est donc pas celui qu'a connu da Silva. 
letras, nað sò se aquieta (come pudera) pedindo a V. M. me enriqueça de cartas suas, passa tambem a pedir com todo o affecto queira V. M. repartirnos desses excellentissimos partos dos ingenhos de Italia, que em Roma se forem publicando: porque em seu cambio serei fidelissimo commissario, retornando tudo o que por aqui se descobrir digno de que $\mathrm{V}$. M. veja, e là se publique. Guarde Nosso Senhor a V. M. muitos anos \&. Castello de Lixboa, 23 de Mayo de r65o.

Pour clôre enfin cette série de témoignages infiniment honorables pour celui qui en fut l'objet, il ne me reste plus qu'à citer quelques lignes du grand savant Isaac Vossius. L'éloge est bref, mais il n'en vaut pas moins'

,Accepit Domina nostra ${ }^{2}$ ante biduum catalogum accuratissimum librorum bibliothecae Althaemsianae ${ }^{3}$. Non possum tibi exprimere quanta cum voluptate illum perlegerim, adeo is multos' et rarissimos continet libros. Procuravit hunc Dominae nostrae Vincentius Noghera, qui $\&$ ipse brevi quoque se transmissurum promittit catalogum librorum Farnesianorum. Noghera autem is est Eques Lusitanus, a multis mihi jam notus annis, vir probus, doctus et Dominae nostrae studiosissimus. Habitat Romae in aedibus, ut puto, Barberianis."

D. Vicente ne revit pas sa patrie; il mourut à Rome, en 1654, au milieu de ces lettrés qui l'avaient si bien accueilli plus de vingt années auparavant et qui surent adoucir son long exil. Un ami italien lui éleva un tombeau et y fit graver l'épitaphe que Barbosa Machado a transcrit à la fin de sa notice.

L'aperçu très sommaire des anciennes littératures catalane et castillane, augmenté de quelques informations sur diverses notabilités littéraires, scientifiques ou artistiques, que Noguera a décoré du titre de Discours sur la langue et les auteurs d'Espagne, ne doit pas être considéré comme la quintessence de longues et profondes recherches, ni même comme un travail de vulgarisation, composé d'après un plan bien arrêté. Ce sont de simples notes, que le correspondant de Peiresc a réunies à la hâte et groupées de son mieux, sans rien faire de plus que d'interroger ses souvenirs. Il avoue lui-même qu'il a tout écrit en une nuit et que sa mémoire a dù faiblir de temps à autres. En effet, les petites erreurs de noms et de dates sont fréquentes, et l'annotatcur de ce discours, quelque désir qu'il ait de ne pas faire parade d'unc érudition trop facile, sera tenu de rectifier certains lapsus. Il faut le dire aussi, l'intérêt de ce chapitre d'histoire littéraire espagnole n'est pas, à

' Burmannus (P.), Sylloges epistolarum a viris illustribus scriptarum, Leyde, $1727, t$. III, p. 627 . La lettre, datée du 23 septembre 165 I, est adressée à Nicolas Heinsius.

${ }^{2}$ Christine de Suède.

3 Cette belle collection, formée par Jean Ange, premier duc d'Altaemps $(+1620)$, fut dispersée à la fin du XVIIe siècle. Mabillon en vit encore une partie en 1685 , a Rome: „Bibliothecae Altaempensis libri editi magna ex parte distracti; manuscripti adhuc venales ad duo millia." Iter italicum litterarium annis $M D C L X X X V$ \& $M D C L X X X V I$, p. 78. 
part quelques détails curieux, dans les faits qu'on y trouve et que l'on connâît d'ailleurs, mais dans la manière dont ces faits sont présentés, dans l'appréciation qui en est donnée. Ces jugements. qui parfois répugnent à notre manière actuelle d'envisager les diffërentes phases d'un développement littéraire, ont leur prix: ils nous ouvrent un jour sur les procédés de critique de ces lettrés du XVII ${ }^{\circ}$ siècle, dont on médit peut - être trop, sans les connaître assez. Nous en savons plus long qu'eux sur beaucoup de choses, en particulier sur cette ancienne littérature, qu'ils regardaient comme une sorte de barbarisme et que nous apprécions plus sain€ment; notre esthétique littéraire a renoncé aux puériles formules que nos pères tenaient pour les règles fondamentales de l'art et du goût, mais cela ne veut pas dire que nous n'ayons pas aussi nos préjugés et nos manies. Ces critiques d'un autre âge avaient du bon, quoiqu'on dise, et c'est dépasser la mesure que de ne voir dans le XVII e siècle espagnol qu'une époque de complète perversion morale et intellectuelle. Il y eut en tous cas dans cette décadence de nobles exceptions. Des hommes comme Quevedo, Pedro de Valencia, Cascales, et bien d'autres qu'on pourrait citer, quelles que soient les concessions qu'ils aient pu faire sur certains points au mauvais courant, ne méritent pas moins d'être considérés comme les continuateurs de la grande tradition du $\mathrm{XVI}^{\mathrm{e}}$ siècle. Ils ont en mainte occasion courageusement défendu la cause des fortes études et du bon goût. Noguera, par son tempérament et son éducation, appartenait à ce petit groupe d'élite, et la doctrine littéraire et scientifique qui apparaît dans la partie critique de son discours est bien la même doctrine que ces maîtres ont préconisée dans leurs écrits.

Le mémoire de Noguera ne met pas seulement en évidence les qualités intellectuelles de son auteur, il peint encore son caractère, si je ne me trompe, sous un jour assez favorable. L'émigré portugais, transplanté dans un monde nouveau, qui le recevait un peu comme une victime de l'étroitesse d'esprit de ses compatriotes, l'émigré portugais n'a nulle part cédé à la tentation de venger ses infortunes personnelles en dénigrant sa patrie. Sans doute il se croit permis de décocher en passant un trait aux abominables falsifications historiques, dont il rougissait en sa qualité de savant sérieux, ou à la déplorable insousiance de certains grands pour les gloires littéraires de la nation, mais il s'abstient partout ailleurs d'attaques contre ses compatriotes: on ne l'entend pas parler d'este pais et de ses choses avec cet accent de mépris qu'on surprend trop souvent chez les Espagnols à l'étranger. Le ton de son discours est celui d'un homme du monde, légèrement sceptique, qui a passé par les grandeurs sans en être ébloui et voit de haut défiler la comédie humaine.

Maintenant que le lecteur est prévenu de ce qu'il trouvera ou plutôt de ce qu'il ne trouvera pas dans ce discours, je n'ai plus qu'à laisser la parole à son auteur. 


\title{
Copia d'un discurso del Sigr Don Vincente Noguera I SOPRa la lingua E li aUtori di SPagna.
}

\author{
Eminmo e Revmo Sigr e Patron Colmo,2
}

Di una lettera che scrissi al Sigr de Peiresch, dimandandogli una man de libri, hebbi una cortesissima risposta, ritratto veramente dell' animo suo, secondo che in Hispagna, e penso che in ogni parte, lo depinge la fama. E lasciando mille altre gentilezze, restai maravigliato di certi quesiti suoi in materie nostre, che mi mostrano in esse esser informatissimo, e tanto che haverei per assai erudito lo spagnolo che in tal modo discorresse e dimandasse. E sapendo lui la volonta con che io li servirei, pur volse darmi alcun merito appresso V. Emin. assicurandomi che ella si terrebbe obligata di tutto il servitio ch'io in ciò li faria. Pero io, havendo inteso anco per altra banda che V. Emin. haveria gusto grande di sodisfare copiosamente al sudetto Signore, mi risolsi di scriver questa informatione intorno li proposti quesiti, per dare con quella qualche pegno della mia divotissima servitù a V. Eminenza. Sono dunque i quesiti:

I. Della lingua spagnuola e catalana e della somiglianza loro con la latina. A che rispondo essergli la castigliana tanto vicina che si trova in essa ciò che in nissuna altra volgare, \& è che ${ }^{3}$ una istessa oratione spagnuola, senza cangiamento di una lettera, ne pur d'un apice, fa il medesimo significato nella latina." Cosa incredibile, si non si videsse stampata la prova in un certo libro chiamato Las obras del Maestro Oliva, rettor de la Vniversidad de Paris, publicadas por su sobrino el cronista Ambrosio de Morales." Qui legga V. E. un discorso"i insieme latino e spagnuolo, che noi chiamiamo romance per deduzzione della lingua romana. E si V. E. puo rubbar al publico una mezza hora di piu, veda nel fine di questo libro un

1 Ms. „Noguerra“.

2 Francesco Barberino.

3 Ms. "che in".

- Bien des érudits se sont exercés à écrire des vers ou de la prose en latin-espagnol. Tícknor (éd. Julius, t. II, p. 455, note 2) a cité un certain nombre de ces tours de force. Voyez encore la Misceldnea de Zapata, p. 308 (Memorial historico español, t. XI). Des phrases ou des vers isolés, tels que le fameux début de Juan de Mena, Canta tu, cristiana musa, ou cet autre vers d'un inconnu, Maria assa tres sardinas, pourraient être recueillis en grand nombre.

5 Voici le titre exact de cet ouvrage: Las obras del maestro Fernan Peres de Oliva, natural de Cordova: Rector que fue de la Universidad de Salamanca y Cathedratico de Theologia en ella. Con otras cosas que van añadidas, como se dara razon luego al principio. Dirigidas al illustrissimo Señor el Cardenal de Toledo, don Gaspar de Quiroga. Con privilegio. En Cordova por Gabriel Ramos Bejarano. Año 1586. Pour donner une preuve de son habileté, Ambrosio de Morales, neveu de l'auteur et éditeur de ses œuvres, a inséré dans le livre une lettre à $\mathrm{D}$. Juan d'Autriche en latin-espagnol, qui commence ainsi: "Si de paterno exemplo (o inclyta potencia de Austria) te incitares..." Noguera donne à tort à Perez de Oliva le titre de recteur de l'université de Paris; ce savant étudia et enseigna à Paris, mais il ne fut jamais recteur qu' a Salamanque.

: C'est le dialogue entrc Siliceo, les mathématiques et la Renumée. 
altro discurso Del disprezzo della morte', composto per un altro terzo autore, ma che messero per maraviglioso nell' assonto, come sempre fu stimato da valenthuomini. E che questa lingua di hoggi si parlasse in Spagna al tempo di Augusto si ingegna di mostrare Gregorio Lopez Madera nei Discorsi sopra le lamine del Monte Santo di Granata :; ma è pazzia di creder ché una di quelle in finissimo spagnuolo, dove si prognosticano le sette Mahometana e Lutherana sia legitima e genuina dell' Apostolo et Evangelista S. Giovanni, scritta cosi, non solo perche havea da publicarsi in questo secolo, ma ancora perche in quello era lingua vernacula \& usata in Spagna.

Della lingua catalana, che volgarmente si chiama limosina, dirò ch’è quella che si parla hoggi in tutto il principato di Catalugna $\&$ in tutto il regno di Valenza e quella che parlavano tutti i Re di Aragona, dal matrimonio del principe Ramon Berenguè con la regina Petionilla, fin a Don Ferdinando Catholico, ultimum Hispanorum ${ }^{3}$, inclusive. E ciò che mi maraviglia è che l'infante di Antequera, avo suo, che fu eletto re d'Aragona in Caspe, con haver regnato solo cinque anni ${ }^{4} \&$ esser castigliano, dal primo anno non scrivea ne parlava altra lingua che la limosina, come si vede in tulte le lettere vicendevoli da lui a Sant Vincenzo Ferrer. ${ }^{3}$ Et hoggi tutte le provisioni reggie, ancor che fatte et sottoscritte da vicere castigliani, sono scritte in limosino.

Ha questa lingua parecchi libri stampati modernamente ma di nessuna importanza. Pur chi havesse i sei seguenti, haveria quanto ci è di prezzo e stima appresso $\mathrm{i}$ dotti di Spagna. I 0 Historia di Ramon Montaner. Tratta dei fatti de' Catalani in Grecia, dove lui si trovò presente a quelle prodezze \& incredibili temerita. ${ }^{\circ}$ Questa istessa historia scrisse e stampò ultimamente don Francesco di Moncada, conde de Ossona, marchese di

1 „Discurso del licenciado Pedro de Valles, natural de Cordova, sobre el temor de la muerte y el amor y desseo de la vida y representacion de la gloria del cielo", f. $228-253$.

2 Discurso sobre las laminas, reliquias y libros que se an descubierto en la ciudad de Granada este año de 1595. Y las reliquias y prophecia que se ari: ha!lado el a.̃o passudo de i588. In foliu de $58 \mathrm{ff}$. nun chiffués, sans frontispice. En tête du livre est un plan gravé qui porte ce titre „Este es el retrato del Monte Santo questa junto á la ciudad de Granada." Plus tard l'auteur reprit le meme sujet et le développa dans ses Discursos de la certidumbre de las reliquias descubiertas en Granada desde el año MDLXXXVIII hasta el de $M D X C V I I I$, Granada, Sebastian de Mena, I60I, in fo. Sur ces écrits et leur auteur, voy. José Godoy Alcántara, Historia critica de los falsos cronicones, Madrid 1868, p. 107 note et 163.

${ }^{3}$ Par ultimum Hispanorum Noguera entend, je pense, le dernier roi d'Espagne qui ait porté le nom de Ferdinand.

- Ferdinand Ier d'Aragon ne règna que quatre ans, du 3 septembre r 4 I 2 au 2 avril 1416; cf. Bofarull, Los condes de Barcelona, t. II, p. 307-309.

' N. Antonio (Bibl. hisp. vetus, t. II, p. 207) et Ximeno, (Escritores del reyno de Valencia, t. I, p. 29) ne parlent que d'une lettre de Vincent Ferrer au roi Ferdinand, et cette lettre fut écrite en latin.

- Il est à peine nécessaire de rappeler que la chronique de Muntaner ne traite pas seulement de l'expédition de Morée, mais de l'histoire des rois d'Aragon depuis Jacques le Conquérant jusqu'a Alphonse III. 
Aitona, opera assai polita \& elegante.' - $2^{\circ}$ L'Historia di Tomich', che tratta de' re antichi \& è assai anticha, pur la fece tradure, ma malamente, il duca di Sessa. ${ }^{3}$ - La $3^{\text {a }}$ è l'Istoria di Carbonel. ${ }^{4}$ Fu scritta intorno i tempi di Ferdinando Catholico. E vasta, ma ha inchiuso un bellissimo pezzo, che sono i commentari del re Don Pedro il $4^{\circ}$, detto del' Pugnaletto, scritti dall' istesso rè, che fu grandissimo politico e niente inferiore al Machiavello. ${ }^{5}$

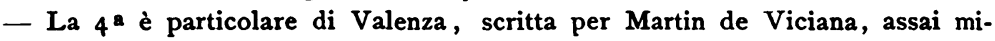
nuta \& util alle genealogie. ${ }^{6}$ Non annovero tra queste quella di Beuter, perch'era schiocco e seguita affatto le favole d'Annio Viterbiense con $i$ suoi Berosi, Manethoni, etc. ${ }^{7}$ - Il $5^{0}$ autore è il poëta Ausias March, che, come Il Decamerone si tiene per la regola del toscano, cosi questo del limosino. Boscan, celebre poëta castigliano, lo tradusse, ma senza lode. ${ }^{8}$ Questi cinque

1 L'Espedicion de los catalanes y aragoneses contra turcos y griegos por don Francisco de Moncada, conde de Osona, Barcelone, 1623, in $4^{\circ}$.

2 Historia e conquestes dells comtes de Barcelona y reis d'Aragó, Barcelona, 1495, in $\mathrm{f}^{0}$. Voy. la description de cette édition princeps dans Mendez, Tipografía española, éd. Hidalgo, p. 330.

$3 \mathrm{La}$ Bibliothèque nationale de Madrid possède trois exemplaires d'une Suma de las Crónicas de Aragon y principado de Cataluña de Mossen Pedro Tomich, traducidas del lemosin al castellano por Fuan Pedro Pellicer (G I 5 I, 152 et 153). Il résulte d'une note du généalogiste José Pellicer, insérée au commencement du ms. G 151 , que ladite traduction fut dédiée à Charles-Quint l'an 1530; d'autre part la première initiale du livre est ornée des armes de Sesa, ce qui pourrait indiquer que le duc prit l'initiative de ce travail et le fit présenter à l'empereur. Je dois ces renseignements à l'amitié de D. José Maria Octavio de Toledo.

1 Chroniques de Espanya fins aci no divulgades... Compilada per lo honorable $y$ discret mossen Pere Miquel Carbonell, Barcelone, 1547, in f'. L'exemplaire de cette édition que possède la Bibliothèque nationale de Paris a appartenu à Gerónimo Zurita, comme l'indique cette note inscrite sur le premier feuillet: „De Auladey y el uso del Sor Jeronimo Çurita." Cet exemplaire était du nombre de ces trente-trois livres d'histoire, dont l'illustre historien se réserva l'usufruit, après avoir fait donation de sa bibliothèque au couvent d'Aula Dei de Saragosse; voy Uztarroz et Dormer, Progresos de la historia en el reino de Aragon, éd. de 1878, p. 118.

- Les Commentaires du roi Pierre IV d'Aragon ont été réimprimés par D. Antonio Bofarull d'après le texte de Carbonell: Crínica del rey de Aragon, D. Pedro IV el Ceremonioso ó del Punyalet, escrita en lemosin por el mismo monarca, traducida al castellano y anotada. Barcelone, 1850 . Les deux mss. de cette chronique que possède l'Académie de l'Histoire a Madrid (voy. le Diccionario de Tomas Muñoz) pourront servir un jour à donner une édition critique de cet important ouvrage.

G On connaît trois livres de cette chronique de Valence, le deuxième, le troisième et le quatrième, qui ont été publiés à Valence en 1564 et à Barcelone en 1566 (pour le détail voy. Pedro Salvá, Catdlogo de la biblioteca de Salvá, No. 3219). Le premier livre, qui a existé, puisque l'auteur le cite, semble perdu.

7 Primera part de la historia de Valencia que tracta de les antiquitats de Espanya y fundacio de Valencia, Valence, 1538, in fo. - Segunda parte de la Crónica general de España, y especialmente de Aragon, Cataluña y Valencia, Valence, 1551 , in fo. Les deux parties ont été réimprimées en castillan à Valence en 1604. Il existe une troisième partie manuscrite; voy. le Diccionario de Tomas Muñoz.

8 Boscan n'a pas traduit Auzias March, mais il l'a beaucoup imité ; voy. Revue critique du 8 avril 1876, p. 243. 
libri si deono pigliar tutti della prima stampa in forma e lettera gottica e si troveranno in Barcelona.

Lasciai per ultimo il megliore di tutti, che, manuscritto (poiche mai non si stampò), sarebbe un ornamento di ogni nobile libraria: e sono i Commentari del Re Don faime il primo', che pigliò dai Mori il regno di Valemza e l'isola di Mallorca, e fu quello che perseguitò a S. Raimund Pennafort, perchè, essendo confessor suo, non volse assolverlo, perche non discedebat a concubina. Scritti dall' istesso re e scritti assai fidelmente, perche schiettamente racconta tutti i suoi falli $\&$ in quante schiagure lo messero li furori della gioventu. In castigliano scrisse l'istoria di questo re diffusamente Miedes, obispo di Albarrazin ${ }^{2}$, ma non è in niente da paragonarsi. E questo è quanto mi occorre del limosino.

Quanto al canzoniero castigliano: fiorirno una man de poëti sotto i tre re, padre, figliuol e nipote, Henrico 3, Giovanni 2, Henrico 4, ma di nessuna arte e di nessuna eruditione, eccetto Juan de Mena sub foanne $2^{\circ}$, che fu un altro Dante, et è stato commentato da quattro valenthuomini ${ }^{3}$, ma il meglior [commentario] di tutti è quello del Pintiano*, chi scrisse pur in Pomponium Melam.5 Di quelli suddetti poeti si stamparono in foglio in lettera gothica, penso che in $\operatorname{Logroño}^{6}$, alcuni versi col nome di Cancionero. E doppo si stampò in Anversa per Joan Stelsium in $\mathbf{8}^{\circ}$, ma molto inferiore dell' altro. Io l'hebbe tutti duoi da un inquisitore, poiche loro l'hanno pro-

- Noguera ne connaissait pas, parait-il, l'édition de ces soi -disants commentaires publiée à Valence en 1557, sous le titre de Chronica o commentari del gloriosissim e invictissim rey en Iacme per la gracia de Deu rey de Aragon, de Maltorques e de Valencia. On a beaucoup discuté sur l'authenticité de cette chronique, mais sans résultat, parceque les éléments d'une étude critique de la question n'ont jamais été réunis. Il est clair que le texte de la Cronica, tel qu'il nous est présenté par l'édition de Valence ou le manuscrit de Poblet (publ. par M. Aguiló y Fuster dans sa Bibliotheca catalana), ne peut pas avoir été écrit par le roi lui-même; ce qu'il s'agit de savoir c'est si ledit texte a été rédigé, oui ou non, à l'aide de mémoires dictés par Jacme. J'ai fait voir il y a six ans (Rivista de filologia romanza, t. I, p. 125-127) qu'il était indispensable d'établir un texte de la chronique d'après tous les manuscrits qui nous restent (aux six que j'ai énumérés on peut encore en ajonter un septième de la bibliothèque particulièze du Roi à Madrid) et de déterminer la valeur exacte de la version latine de Marsilio. Depuis lors, à ma connaissance du moins, la question n'a pas fait un pas. Dans l'article de la Rivista, dont les épreuves ne m'ont pas été remises, se sont glissées plusicurs fautes; je profite de l'occasion pour les corriger: P. 126, col. 1, 1.6: Cesar augusta lisez Cesaraugusta; ibid. 1.17 du bas: Quadrado lisez Gallardo; ibid. 1.9 du bas: outre lisez contre; p. I27, col. 1, 1. 14 du bas: partida lisez nostra partida; p. 127, col. 2, 1. 17: biografiques lisez biographiques.

- De vita et gestis Facobi primi regis Aragonum, Valence, 1572, in $\mathrm{fo}^{\circ}$ Une traduction de ce livre fut publiée à Valence en 1584 , in $\mathrm{f}^{\circ}$.

3 Je n'en connais que trois: Hernan Nuñez de Toledo, Hernan Nuñez de Guzman et Francisco Sanchez, el Brocense.

- Hernan Nuñez de Guzman, el comendador griego.

s Castigationes in Pomponium Melan, 1543, in 80.

- Je ne sais sur quel fondement repose cette conjecture de Noguera. Le premier Cancionero general, celui de Juan Fernandez de Constantina, ne porte sur son titre ni lieu ni date, mais on croit qu'il a été imprimé à Valence (voy. F. Wolf dans Ticknor, éd. Julius, t. II, p. 529, note 3); le second, de Fernando del Castillo, est daté de Valence 15 II et a été réimprimé souvent mais jamaris, que je sache, à Logroño. La première édition d'Anvers est de 1557 . 
hibito affatto, bastando scastargli le parodie di Garci Sanchez de Badajoz delle 9 lettioni dell' ufficio dei défonti all' amor profano:

Parce mihi, domine; nihil enim etc.

Perdoname, amor, amor,

Que mis dias no son nada,

Pues al fin de mi jornada

Me tiene tu disfavor.

Dime qué cosa es el hombre,

Pues que tanto le engrandeces....

Doppe qual profanamento il detto Garci Sanchez, come di Theopompo si racconta, si impazzi, et al fine si ammazzo con le proprie mani.

Poco è quello che si stampò nel Canzionero a rispetto di cio che va manoscritto, ma non è cosa per la quale, e gia li megliori pezzi messe il divino Fernando di Herrera nel Commentario sopra Garcilasso2, e, tra li altri, il primo sonetto che si fece in Spagna, e fu quello del Marchese di Santillana ${ }^{3}$, padre del primo duca del Infantado e del cardinal di Toledo, Don Pedro Gonzalez di Mendoza, e di quelli celebri fratelli 4, \& è assai elegante pro illa aetate. Io vidde assai pezzi nell' Escuriale; e ci sono le poësie del re Alfonso 1o, l'Astronomo, eletto re de' Romani, scritte in lingua portoghese, quae tunc in deliciis erat, ma non meritano la fatica di copiarle. 5

Prosegue il sigr de Peiresc dimandando il Santo viaggio ${ }^{6}$ di Ambrosio di Morales se si troverà e dove, et anchor che in doe parole categoriche mi sbrigarei del quesito, pur mi distenderò assai, perche so che haverà piu sodisfattione di alcune noticie incontraticie che della principale.

Ambrosio di Morales, nato in Cordova de padri hidalghi (questa è mezza nobiltà tra l'equestre e la plebe, e pur ha un grande vantaggio che è immemoriale; fatta solamente da Iddio non da' re, i quali non possono far un hidalgo, ancorche possino far e fanno cavaglieri e duchi), fu da giovene santissimo e dottissimo. Pur si fece frate di S. Gerolamo ${ }^{7}$, dove era un

1 Ms. ,ingrandezco." Cf. l'éd. d'Anvers, 1557, f. CLXI ro.

- Obras de Garci Lasso de la Vega con anotaciones de Fernando de Herrera, Séville, 1580 , in $4^{\circ}$. Les vers cités par Herrera appartiennent presque tous à des poètes de la nouvelle manière italienne, on n'y trouve pour ainsi dire rien de l'ancienne école castillane.

3 C'est le sonnet "Lexos de vos e çerca de cuydado." Voy. le commentaire de Herrera, p. 80, et les Obras de Don Inigo Lopex de Mendosa, marqués de Santillana, éd. Amador de los Rios, Madrid, 1852, p. 283.

1 Le premier marquis de Santillana eut sept fils, que nous énumérons dans l'ordre que leur assigne Lopez de Haro dans son Nobiliario genealógico: I. Diego Hurtado de Mendoza, $2 e$ marquis de Santillana, créé duc de l'Intantado par les Rois Catholiques en 1475 ; 2. Iñigo Lopez de Mendoza, I er comte de Tendilla; 3. Lorenzo Suarez de Mendoza, I er comte de Coruña; 4. Pedro Laso de Mendoza, seigneur de Mondéjar; 5. Pedro Gonzalez de Mendoza, archevêque de Séville et de Tolède, et cardinal; 6. Juan Hurtado de Mendoza, seigneur del Colmenar; 7. Pedro Hurtado de Mendoza, adelantado de Cazorla.

5 Il s'agit ici des fameuses Cantigas, dont on ne possède pas encore d'édition complète. L'Academia Española en prépare une depuis un temps immémorial.

i Voy. plus bas.

${ }^{7}$ Morales fit profession en 1533 au couvent hiéronymite de Valparaiso près Cordoue; voy. sa biographie par le P. Enrique Florez en tête du Viage 
specchio de ogni virtì e scienza, \& havendo consummatosi nella theologia et essendo grande predicatore, li venne una volta una tentatione sensuale della carme, e per non consentirla e mortificarsi, mettendo $i$ testicoli dentro in una arca aperta, col coperchio se gli tagliò afatto.' $\mathrm{Ma}$ doppo esser curato dai fratii e guarito quasi per miracolo, per esser allora d'anni quaranta ${ }^{2}$, lo licenziorno, ma subito lo pigliò per chronista suo Philippo 2 con grossissimo salario ${ }^{3}$, e lui sene andò ad habitar in Alcala de Henares, academia, 15 miglia da Madrid, dove allevo in casa sua, mentre visse, 30 gentilhuomini illustrissimi, che sotto la sua disciplina, come d'un altro Origenes, riuscirno eccellenti, et è da notare che, essendo bello e barbato, subito doppo che si castrò li cascò la barba, segli infemini la voce e la faccia segli arrugo, in modo che restò bruttissimo, piu simile a simia che ad huomo, pur un oraculo di Spagna, perche senza controversia era il primo et anco primus Hispanorum, qui in historia nugari desizt. La sua si puo legger come un evangelio humano, giudicio esquisito, grande noticia delle antichità romane, e che in un foglio dice piu \& insegna piu che Lazio ${ }^{5}$, Rosino ${ }^{6}$, Demstero ${ }^{7}$ in dieci, grande pietà che si scorge e spira in ogni parola. Come Philippo 2 lo hebbe in gran credito, lo mandò con grossa spesa e facoltà grandissime a visitar tutte le reliquie e archivi delle cathedrali et abbatie di quella corona, et egli lo fece con grandissima sodisfattione, e si fusse vissuto piu, partorirebbe di grandi effetti. Pur questa peregrinatione con bellissime narrationi messe in un libro che pensava stampare col nome del Santo Viaggio.

E qui è bisogno far una attinentissima digressione, dicendo a V. E. chi è Don Thomaso Tamayo di Vargas, nella cui mano hoggi in Madrid si trova questo libro originale. $^{8}$ Questo è un gentilhuomo toletano richissimo, perche gli fece immense donationi un zio suo, che fü generale degli Osservanti, vescovo d'Osma e Pamplona, chiamato frà Mattheo di Burgos. Fù il Tamayo discepolo charissimo del celebre jesuita Mariana, e tanto charo que l'apologie che il Mariana scrisse in diffesa della sua historia, volse che uscissero col nome del Tamayo", che è buonissimo theologo, buon filosofo, buon huma-

de Ambrosio de Morales por orden del rey $D$. Phelipe II d los reynos de Leon y Galicia y principado de Asturias, Madrid, 1765, in $\mathrm{fo}$.

'Ce! acte d'hérö̈sme, rajporté déjầ par de Thou (Histcricrum, li\%. 99, $\S$ XVII, éd. de Londres, I733, t. IV, p. 899), qui le tenait peut-être de Noguera, est confirmé par le P. Florez.

2 Il quitta le couvent peu après 1535.

${ }^{3}$ Le titre de cronista del Rey lui fut déjà donné par Charles Quint; voy. Florez, l. c., p. XI.

- La coronica general de España et Las antiguedades de las ciudades de España, qui font suite aux cinq livres de la Coronica general de Espanta de Florian de Ocampo.

5 Wolfgang Lazius, médecin et historien allemand, mort en 1565.

6 Jean Rosin, antiquaire allemand, mort en 1626.

7 Thomas Dempster, érudit écossais, mort à Bologne en 1625 .

8 Plutôt une copie de l'original. La Bibliothèque nationale de Madrid possède (d'après l'indice copié par Gallardo) un „Viaje santo, copiado del original, segun dice Tamayo de Vargas en una nota al principio" (X, I7), qui est probablement le ms. dont parle ici Noguera. L'original se trouvait a l'Escurial du temps de Florez.

- Historia general de España del P. D. Iuan de Mariana defendida por el Doctor Don Thomas Tamaio de Vargas contra las advertencias de Pedro Mantuano, Toledo, 1616 , in $4^{\circ}$.

Zeitschr. f. rom. Ph. 111 . 
nista, veramente un rarissimo soggetto, si oeconomiam excipias. Hà stampato una centena de libri, mà da essi si può dir ciò che dalla terra d'Egitto: $\pi 0 \lambda \lambda \dot{\alpha} \mu \dot{\varepsilon} \nu \varepsilon \sigma \vartheta \lambda \dot{\alpha}, \pi 0 \lambda \lambda \alpha \delta \dot{\varepsilon} \lambda v \gamma \rho \dot{\alpha}$. Ultimamente publicò con grande apparato e commentarij la historia di Flavio Dextro per autenticarla. ${ }^{1}$ Et bene audit apud meos Hispanos, perche in somma pro talibus labris tales lactucae. Il presente rè subito lo fece suo chronista di Castiglia con commodissima provisione. Questo gentilhuomo possiede assai manoscritti con intento di publicargli. ${ }^{2}$ Era grandissimo amico mio e volse stampar questo Santo Viaggio e dedicarmelo, e consigliandoli io à chi sarebbe meglio, mai volse mutarsi ; anzi me scrisse in aprile de $16_{3} 1$ che già era d'accordo col stampatore che cominciasse l'opera nel primo di quel luglio e che già havea la charta, che erano 80 risime e gli costarono cinquanta scudi. Io gli mandai subito, e lui nolens volens gli accettò e ringratiò, mà avanti al termino di cominciarsi fui io fatto prigione in $17 \mathrm{di}$ giugno. Domandai, postquam effugi crudeles terras, se si stampò, e mi dicono di nò, anzi che fù tanto il suo disgusto che affatto lo lasciò. Se Monsigr Nontio ${ }^{3}$ gli domandasse vna copia, è tant' ingenuo e facile che non se la negaria. ${ }^{4}$ Io non viddi mai questa opera, mà bastami saper l'autore per reputarla compitissima. Altre parecchie richezze manuscritte hà questo gentilhuomo e sempre grandissima strettezza de danari, e per niente le daria, mà è tale l'inertia di quelli miei signori, che standoli un grande che da molti anni in qua dà 500 scudi al mese ad una cortigiana, non darà per un singolarissimo manoscritto 500 quatrini. Tanta è la virtù di quella corte!

Finisce il sigre de Peiresc (al manco in ciò che della lettera à V. E. me mostrò il conte Ubaldini) ${ }^{s}$ trattando dei manoscritti che havea e mette nel suo indice Gonçalo Argote de Molina. ${ }^{6} \mathrm{E}$ perche di questo celebre scrittore si hà pocha notitia ancora in Madrid e mi la diede minutissima il Sigr. cardinale de la Cueva ${ }^{7}$, compatriota suo (tutti dui sono di Ubeda), nel tempo che non usciva di casa mia e la fissai troppo nella mia memoria, la dirò liberamente, e più libera perche non hà lasciato descendenti, à chi pregiudichino diffetti, in che lui non havea culpa veruna.

1 Flavio Lucio Dextro, caballero español de Barcelona, prefecto-pretorio de Oriente por los años del Sor de CCCC. Defendido por Don Thomus. Tamaio de Vargas. Madrid, 1624, in $4^{\circ}$.

${ }^{2}$ Les nombreux livres et manuscrits que Tamayo avaient réunis lui servirent à rédiger sa funta de libros, la mayor que España ha visto en su lengua hasta el año 1624 (un exemplaire ms. à la Bibl. Nat. de Madrid, If, 23 et 24), premier essai, je crois, d'une bibliographie générale espagnole. Nicolas Antonio s'en est beaucoup servi.

${ }^{3}$ Lorenzo Campeggio, évêque de Sinigaglia, d'après D. Vicente La Fuente, Historia eclesiástica de España, t. VI, p. 449.

Il y a lieu de croire que le cardinal Barberino demanda une copie du Viage santo et l'obtint, car Nicolas Antonio dans sa Bibliotheca nova (s. $\therefore$. Morales) parle d'un ,exemplum huius operis transcriptum ex alio bibliothecae Barberinae."

s Cf. plus haut, p. 19.

- La liste des sources qui se trouve au commencement de la Nobleza del Andaluzia.

7 Alonso de la Cueva, premier marquis de Bedmar, connu dans l'histoire sous le nom de cardinal de la Cueva. 
Morse Argote in Sevilla d'anni 42 senza figliuogli ne anco haver hereditato i duoi contati di Gomera e Lanzarote, due isole Canarie, per haverglo sopravissuto il conte suo socero. Era signore della nobile torre de Gil de Olyd, mà pativa infamia, che uno degli otto bisavi suoi era discendente di hebreo converso alla fede christiana, e come questo mancamento in Spagna è perpetuo et inhabilita in aeternum, volendo ciascuno di noi anzi esser sportaruolo o facchino che duca con quella machia (e pur si trovano ducchi con essa) ' , lui, che era ingegnosissimo e spiritoso, si messe ad imaginar come potria uscir da questo fastidio, e vedendo che solamente con istromenti si vincono simili difficoltà, si messe ad ammassar tanti che è una maraviglia. Et in ordine à mostrar che certa croce decussata $X$ (che in Spagna chiamiamo aspa, et è quella del martyrio dell' apostolo S. Andrea), con che stava dissegnato quel ascendente suo, non era insegna infame chiamata sanbenito ó saccus benedictus ${ }^{2}$, con che gli Inquisitori puniscono $\mathrm{i}$ heretici, ma segno che pigliarono gli conquistatori della città d'Ubeda, tutti i quali la messero variamente in un arco di certa chiesa, fece quel grande libro delle nobilezze ${ }^{3}$ del Andalusia, che si fusse continuata e non restasse smezzata, sarebbe opera illustrissima; pur in questo arco è apresso i suoi paesani modicae fidei, nel resto verissimo, giudicioso et essatto. Havea pensiero d'arrichir Spagna de' suoi manoscritti, e havea già publicato duoi assai notabili, di che darò breve raggunglio, perche sono degni d'haver luoco in ogni seletta libraria. Chiamasi [il primo] Il conte Lucanor 4, libro morale di novelle et historie vere in lingua spagnuola antiqua. Autore fù l'infante Don Juan Manuel, figliuol del infante D. Manuel e nipote del rè Don Fernando il Santo, che pigliò da Mori Sevilla e Cordova, cugino del rè San Luigi, figlio S. Luigi della maggiore delle sorelle D. Bianca e Don Ferdinando $3^{\circ}$ figlio della più giovine $\mathrm{D}$. Berenghela. ${ }^{3}$ Questo $D$. Juan Manuel hebbe due figlie regine, di Castiglia

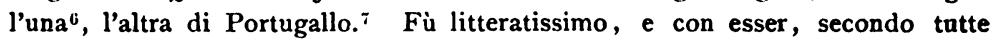
l'historie, assai inquieto e che sempre diede fastidij à $i$ rè nepoti e generi suoi, con tutto ciò osservando la dottrina, che poi spiegò il Macchiavello, nessuna parola si trova in questo libro che non spiri pietà, verità, disprezzo

1 Vuir à ce sujet ie fameux pampnilet de Francisco de Mendoza Bovadilla, intitulé El tizon de España.

2 On peut voir dans le tome $\mathrm{V}$ des Dellices de $l$ Espagrne et du Portugal par Alvarez de Colmenar une gravure du sambenito avec la croix de S. André, insigne des pénitens qui confessaient leur crime avant d'être jugés.

${ }^{3}$ Ms. „nozze".

1 Le Conde Lucanor a été publié pour la première fois à Séville en 1575 et réimprimé quatre fois depuis: la dernière édition est celle de $\mathrm{D}$. Pascual de Gayangos dans les Escritores en prosa anteriores al siglo $X V(B i b l$. Rivadeneyra).

5 Noguera suit ici l'opinion de Mariana touchant la primogéniture de Blanche de Castille, et l'on sait a quelles revendications cette thèse historique a donné lieu. Voir surtout les appendices II, III et XVII des Memorias historicas de la vida y acciones del rey D. Alonso el Noble, octavo del nombre, recogidas por el marques de Mondexar, Madrid, Sancha, 1783, in $4^{\circ}$.

- Jeanne Manuel, mariée à Henri II de Castille.

i Constance Manuel, mariée d'abord à Alphonse XI, roi de Castille, qui qui la répudia, puis à Pierre Ier, roi de Portugal. 
del mondo: in somma leggerlo e legger' il Granata ' 8 Ribadeneira.2 Messe il Argote nel principio del libro una genealogia de tutti $i$ descendenti di questo Infante, mà molto diffettuosa, perche solo in quelli di Castiglia stà compito, non in quelli di Fiandra, di Portogallo, contentandosi di dir che hebbe un figlio chiamato D. Henrico Manuel, conte de Sintra in Portogallo, di cui la restò larga discendenza, mà non esprime, dovendo et potendo arrivar fin' à questo misero relatore. L'altra opera del Argote fù publicare l'originale historia del Tamerlano scritta da i duoi ambasciatori del re di Castiglia Henrico $3^{\circ}$ Payo Gomez de Sottomaior e Ruy Gonzalez de Clavijo. ${ }^{3}$ Libro è che lessi sei volte e sempre con nuovo gusto e maraviglia, perche è il miglior pezzo dell' historia che si trova nel soggetto. Hebbero grande introduttione col Tamerlano, che gli havea alla sua tavola e gli trattava come figliuoli, e loro erano assai acorti e così scrivono sincerissimamente, ma con gran prudenza. E del imperatore di Costantinopli e del rè Ladislao di Napoli scrivono benissimo. In somma è libro picinino mà buonissimo. - Altro publicò assai grande della caccia, scritto dal rè don Alfonso $11^{\circ}$ che vinse la battaglia del Salado, sed nullius frugis. ${ }^{4}$

Havea il Argote assai manoscritti, perche era molto riccho et allhora ancora si stimavano manco che hoggi; mà molti di titolo speciosissimo che lui vidde sono chosa da niente, e. ga quelli tanto nominati 3 tomi della nobiltà de Germania, presentati da Otto Truchses ${ }^{5}$, cardinale, vescovo d'Augusta. Stanno nel Scuriale, dove, saranno venti mesi, gli stetti giorni intieri leggendo et essaminando: ciascun pezzo caricarà una soma di bellissime maniature e pieni di scudi d'arme, pur sciocchissima la narrativa, inordinata, manca, piena d'errori et di contradittioni, et io, con esser hospes in grammaticis, potria far una longa lista di esse, et è molto più importante il Theatro

1 Luis de Granada.

2 Pedro Ribadeneyra.

3 Noguera fait ici une confusion. Henri III de Castille envoya deux ambassades au Tamerlan. La première fut dirigée par Payo Gomez de Sotomayor et Hernan Sanchez de Palazuelos, qui n'écrivirent ni l'un ni l'autre de relation de leur voyage. La seconde, qui eut lieu en 1403 et dont fit partie Ruy Gonzalez de Clavijo, a été racontée par ce dernier, et c'est ce récit que publia Argote de Molina sous le titre de Historia del Gran I'tmorlan e Itinerario y enarracion del viage, y relacion de la embaxada que Ruy Gonçalez de Clavijo le hizo, por mandado del muy poderoso Sentor Rey Don Henrique el Tercero de Castilla, Séville, 1582, in 10; réimprimée par Sancha, Madrid 1782.

- Libro de la monteria que mando escrevir el muy alto y muy poderoso rey Don Alonso de Castilla $y$ de Leon, ultimo (ce n'est plus vrai aujourd'hui) deste nombre. Acrecentado por Gonçalo Argote de Molina, Séville, 1582, in $\mathbf{f}^{0}$.

s Othon Truchses de Waldpurg, évêque d'Augsbourg (1543-1573), créé cardinal l'an 1544. Ce personnage accompagna en Espagne les archiducs Rodolphe et Ernest en 1564;' voy. Gachard, Don Carlos et Philippe II, 2 c éd., p. II 3. Dans l'introduction de la Nobleza del Andaluzia Argote parle de l'ouvrage de Truchses en ces termes: „Escrivieron de linages.... sobre todos Othon, Cardenal de Augusta, quatro libros manuescriptos de excelente iluminacion, que dedicó y presentó al Rey nuestro señor, de la nobleza universal y particular de los Estados Septentrionales"; puis il le cite dans la liste de ses sources: „Oton, Cardenal de Augusta, de la nobleza universal y particular de la de Alemaña." 
genealogico di Girolamo Henninges Luneburg'. Il In $^{n}$ tomo dcl Truchses mette i nove della Fama, à Josue col sole, à David colla harpa et altre simili ciaccere di scudi fictitij quae referre pudet. In summa thesaurus carbones, e se io havessi alla mano la lista d'Argote, forsi darei buona raggione d'una gran mano d'essi.

Quando morse l'Argote non si trovò chi comprasse i suoi libri, medaglie, statue, pitture, se non Luciano di Necrone ${ }^{2}$, genovese, richissimo canonico di Sevilla, al quale lasciò anche Arias Montano ${ }^{3}$ i suoi libri e peculio antiquario. Tutta questa robba me dicono che, morto il Luciano, comprò il duca d'Alcalat $\mathrm{e}$ che la tiene tan chiusa e guardata ac gryphes aurum. Non conosco questo signore, perche in tempo mio non stette mai in Madrid, mà penso che è tan curioso in ammassar come avaro in non prestar, mostrar, lasciar conferire o copiare. F. così siamo fatti.

Mi domanda più il Sigre Peiresc per le giostre del ponte d'Orbygo. Et è un libro di $\mathbf{8}^{\circ}$, che in Madrid si troverà per un testone, chiamato $I l$ passo honroso. 5

Juan de Arfe fù un grande orcice de Sevilla, il primo della sua mechanica, perche havea qualche notitia di architettura $e$ geometria, in che stampd qualche operette 6 , come etandio della custodia del domo de Sevilla, che lui lavorò di mano e bottega sua, la quale pesa di argento diciotto milla scudi e vale più la fattura. Anche apriva sigilli et era qualificato[re] de

1 Theatrum genealogicum, ostendans omnium aetatum familias. Magdebourg, 1598, 7 vol. in $\mathrm{f}^{\circ}$.

2 Je ne sais rien sur ce personnage, qui d'ailleurs ne recueillit pas toute la bibliothèque d'Argote de Molina. Une partie de cette collection échut à D. Garci-Lopez de Cardenas, neveu d'Argote, qui n'en fit rien et n'en prit aucun soin (voy. l'Ensayo de una bibl. esp. t. I, col. 28I), et l'on trouve une liste de mss. ayant appartenu à l'historien andaloux dans un catalogue imprimé s. 1. n. d., dont parle D. Pascual de Gayangos, Escritores en prosa ant. al s. $X V$. p. 231 , note 2.

3 D'après Nicolas Antonio (Bibl. hisp. nova) Arias Montano légua ses inanuscrits à la bibliotheque de l'Escurial et ses livres au couvent de $\mathrm{S}$. Jacques à Séville.

1 D. Fernando Afan de Ribera Henriquez, troisième duc d'Alcalá, mort le 28 mars 1637. Sur sa bibliothèque voy. l'Ensayo de una bibl. esp., t. I, col. 29-30. On trouve cité dans le Museo o biblioteca selecta de el Excmo Señor Don Pedro Nuñez de Gusman, marques de Montealegre, Madrid, 1677 , f. 111 vo, un ,Indice de los libros manuscritos que huvo en la libreria del duque de Alcalá, marques de Tarifa, en fol. MS.".

5 Libro del passo honroso defendido por el excelente caballero Suero de Quiñones. Copilado de un libro antiguo de mano por Fr. Fuan de Pineda. Salamanca, 1588, in $8^{\circ}$. Réimprimé par Sancha, Madrid, $1783,4^{\circ}$.

- Quilatador de la plata, oro y piedras conforme d las leyes reales y para declaracion de ellas. Hecho por foan de Arphe Villafarte, natural de Leon, esculptor de oro y plata, ensayador mayor de la moneda en la real y antigua casa de Segovia, Madrid, 1598, in 80. Réimprimé à Madrid en 1678 , in $4^{\circ}$, avec un autre manuel du même autcur imprimé pour la première fois à Valladolid en 1572. - De varia commensuracion para la sculptura y architectura. Séville, $1585-1587$, in fo. Réimprimé en 1675, à Madrid, in $\mathrm{f}^{\circ}$; en 1736, à Madrid, in $\mathrm{fo}^{\mathrm{o}}$ avec des additions de D. Pedro Enguera, et encore à Madrid très augmenté par José Asensio, en 1806, 2 vol. in fo. Descripcion de la traça y ornato de la custodia de plata de la sancta iglesia de Sevilla. Séville, 1587 , in $8^{\circ}$. 
metalli, perche intendeva tutto questo bene. Nella materia de arme et scudi ${ }^{1}$ niente ${ }^{2}$ lessi in lui che non si trovi piu ben trattato in un libretto francese, che penso si chiama Eloquence francoise par Rene Frangois, predicateur du $R o y^{3}$, dove facendo discorsi in ogni arte, accioche non si sbaglino in parlar d'esse i predicatori, stà un discorso des armoiries, che me parve il migliore e più sostantiale di questo argumento. Habbiamo bene in Spagna il Nobiliario di Fernan Messia stampato nel ${ }_{1} 488$ in fol. ${ }^{4}$, mà è prolisso et inetto, mettendosi à combinare $i$ colori con gli elementi et humori, con altre simili ciaccere.

De materie genealogiche havea io forse più che nessuno, perche erano due grande casse piene di singolarissima materia per la stampa, pur per intenderla e giovarsi sarebbe bisogno dare io la chiave, perche stà quasi in cifera (al manco le verità, che doveano sapersi e non esprimersi), da donde penso che nessuno si havrà giovato molto.

$\mathrm{E}$ questo è, Eminmo Sigre, quanto in una notte che ho denegata al sonno ho potuto cavar dalla memoria, che forsi me haverà gabbato, perche, essendo potenza in tutti debole, in me è debolissima e fallacissima, e cosi, si qualche chosa si troverà in contrario, libens cedo et indictum volo. $\mathrm{E}$ baccio humilissimamente i piedi di V. E. Roma, 5 di gennaro 1637.

Eminmo et Revmo Sigre,

De V. E. R.

obligatissmo serre

Don Vincente Noguera. ${ }^{5}$

1 Argote de Molina cite dans l'indice de sa Nobleza del Andalusia un "Libro de escudos de armas por Iuan de Arfe".

2 Ms. "mensi“, ce qui ne donne pas de sens.

3 Dans l'Essay des merveilles de nature et des plus nobles artifices. Piece tres-necessaire a tous ceux qui font profession d'eloquence. Par René François, predicateur $d u$ Roy, Rouen, 1621 , in $4^{\circ}$, se trouve en effet un traité „Pour blasonner les armoiries des roys, princes, pays, \&.“ (p. 43I-446), précédé de cet avertissement: „Amy lecteur. Il eschet mille fois qu'il faut parler des armes des familles, \& on ne sçait par quel bout commencer. Aux oraisons funebres des grands, aux loüanges des grandes familles, aux receptions des admiraux $\&$ officiers de la Couronne $\&$ en mille autres occasions il est du tout necessaire de parler des armes, mais la faute est d'autant plus lourde qu'elle est faite à la vollée devant une si belle compagnie. Ie vous veux aider à ne faillir point ou peu quand il vous fauldra parler de cette matiere".

4 Libro intitulado nobiliario perfetamente copylado e ordenado por el onrrado cavallero Fernandt Mexia, veynte quatro de Fahen. Séville, 1492, in $\mathrm{f}^{\mathrm{o}}$.

"Ms. „Noguerra“.

A. Morel-Fatio. 